\title{
Consumption-investment optimization problem in a Lévy financial model with transaction costs and làdlàg strategies.
}

\section{E. LEPINETTE and T.Q. TRAN}

\begin{abstract}
We consider the consumption-investment optimization problem for the financial market model with constant proportional transaction rates and Lévy price process dynamics. Contrarily to the recent work in [4], portfolio process trajectories are only left and right limited. This allows us to identify an optimal làdlàg strategy, e.g. in the two dimensional case, as it is possible to suitably rebalance the portfolio processes when they jump outside the no trade region of the solvency cone.
\end{abstract}

Keywords · Consumption-investment Problem - Transaction costs · HamiltonJacobi-Bellman equation · Viscosity solution · Lévy process.

Mathematics Subject Classification (2000) 60G44

JEL Classification G11 · G13

\section{Introduction}

We study a consumption-investment problem with infinite horizon for financial market models including proportional transaction costs and when price's dynamics is driven by a Lévy process. This problem originates from the seminal paper of [2]. Davis and Norman [3] rigorously solved the problem and provided the optimal consumption plan in a diffusion model with transaction costs. Although the value function $W$ is in general not smooth, Soner and Shreve [12] shows that $W$ is solution to the HJB equation in a weak sense, i.e. is a viscosity solution. When the risky asset prices follow exponential Lévy processes, Framstad et al. [6] have obtained the same results as those of [12]

E. Lépinette

Ceremade, CNRS UMR 7534, Paris Dauphine University, PSL National Research, Place du Maréchal De Lattre De Tassigny, 75775 Paris cedex 16, France and Gosaef, Faculté des Sciences de Tunis, 2092 Manar II-Tunis, Tunisia. E-mail: emmanuel.lepinette@ceremade.dauphine.fr .

T.Q. Tran

Ryerson University, George Vari Engineering and Computing Centrer, 245 Church St, Toronto, ON M5B 2K3, Toronto, Canada.

75775 Paris cedex 16, France

E-mail: : tuan.maths@gmail.com 
under some mild conditions. An extension to the jumping diffusion case is studied by Kabanov et al. [4] where a general market model with conic constraints is considered.

The present paper extends [4] to the case where the controls (the portfolio strategies and consumption plans) are only left and right limited (làdlàg). This generalization is important as we need it to identify an optimal strategy, as conjectured in the twodimensional case by Framstad et al. [6]. Indeed, in [6], the set of controls is the family of all right continuous and left limited strategies but the conjectured optimal scontrol is only làdlàg. Actually, despite this contradiction, considering làdlàg strategies is the good intuition as the negative effects induced by price process jumps on portfolio processes may be corrected by an immediate re-balancement. The economic intuition is also straightforward. When asset prices move in response to unpredictable events, which is typically the case at jumping times of a Lévy process, a trading response should be immediately be made after the event i.e. the strategy or control should only be right limited. However, if a market information is announced at a predictable time, e.g. in the presence of a predictable labor income stream, a trade may be executed immediately before the announcement, i.e. the strategy is right-continuous at that predictable time.

Although the main results in this paper extend the results of [4] and [6], one needs to re-examine carefully each step of the proofs. One of the most difficult part is to show that the Bellman function is the unique viscosity solution to the HJB equation. In the presence of one bond and one risky asset, we study the regularity of the Bellman function and give a construction of the optimal strategy which is conjectured in [6].

The paper is organized as follows:

- Section 2: Description of the consumption optimization problem.

- Section 3: Elementary properties of the value function (Bellman function) $W$.

- Section 4: We show that $W$ is a viscosity solution of a Hamilton-Jacobi-Bellman (HJB) equation.

- Section 5: We show that the (HJB) equation admits a unique solution under some mild conditions and as soon as there exists a Lyapunov function.

- Section 6: We propose a condition under which $W$ is finite. This is in particular the case when there exists a non negative classical supersolution to the (HJB) equation.

- Section 7: We show that $W$ is continuous when finite.

- Sections 8,9: For the power utility function, we construct a Lyapunov function and a non negative classical supersolution for Sections 5 and 6 .

- Section 10: We apply the general results to a two-dimensional model. Under some conditions, we prove that the value function is continuously twice differentiable and we construct an optimal control. To do so, we solve a Skorokhod problem. Note that we introduce in this part a new definition of viscosity solution in a weaker sense. This allows us to change the global operator by a local one and then deduce that the Bellman function is $C^{2}$.

- Appendix: Resolution of the Skorokhod problem for Section 10.

\section{Notations:}

In $\mathbf{R}^{d}$, we use standard notations like $|x|$, the Euclidean norm of $x \in \mathbf{R}^{d}, d(x, y)=$ $|x-y|$, etc. The Euclidean scalar product between two vectors $x, y \in \mathbf{R}^{d}$ is denotes by $x y \in \mathbf{R}$.

We shall use the notations $A^{+}$and $A^{-}$to designate the left (resp. right) limit of a process $A$ and we also denote by $A_{t-}$ and $A_{t+}$ its left and right limit at time $t$. If $A$ is 
a làdlàg predictable process of finite variations, the left and right jump processes are denoted by

$$
\Delta A:=A-A^{-}, \Delta^{+} A:=A^{+}-A,
$$

and we introduce the associated càdlàg processes:

$$
A_{t}^{d}:=\sum_{s \leq t} \Delta A_{s}, \quad A_{t}^{d+}:=\sum_{s<t} \Delta^{+} A_{s}, \quad t \geq 0 .
$$

The continuous part of $A$ is defined as

$$
A^{c}:=A-A^{d}-A_{-}^{d,+} .
$$

We denote $\dot{A}^{c}$ the optional version of the Radon-Nikodym derivative $d A^{c} / d\left\|A^{c}\right\|$ where $\left\|A^{c}\right\|$ is the total variation of $A^{c}$.

\section{Optimal consumption investment problem}

We consider the financial market model with jumps adopted in [4]. The price return process is modeled by a $d$-dimensional Lévy process $\left(Y_{t}\right)_{t \geq 0}$ defined on a stochastic basis $\left(\Omega, \mathcal{F},\left(\mathcal{F}_{t}\right)_{t \geq 0}, \mathbb{P}\right)$ satisfying the usual conditions. We denote by $p(d z, d t)$ its jump measure and $q(d z, d t)=\Pi(d z) d t$ its compensator. We suppose that $\Pi(d z)$ is a non negative measure concentrated on $(-1, \infty)^{d}$ and

$$
\int_{\mathbf{R}^{d}}\left(|z|^{2} \wedge|z|\right) \Pi(d z)<\infty
$$

The dynamics of $Y$ is given by

$$
d Y_{t}=\mu t+\Xi d W_{t}+\int_{\mathbf{R}^{d}} z(p(d z, d t)-q(d z, d t))
$$

where $\mu \in \mathbf{R}^{d}, W$ is a $m$-dimensional standard Brownian motion and $\Xi$ is a matrix of dimension $d \times m$. In the identification of an optimal strategy, we shall only consider a pure jump Lévy process with finite activity, i.e. $\int_{\mathbf{R}}|z| \pi(d z)<\infty$. The general case remains open.

Two constant cones $K$ and $\mathcal{C}$ are given. They are supposed to be closed and proper, i.e. $K \cap(-K)=\{0\}$ and $\mathcal{C} \cap(-\mathcal{C})=\{0\}$. We assume that $\mathcal{C} \subseteq$ int $K \neq \emptyset$. In finance $K$ and $\mathcal{C}$ stand respectively for the set of transaction constraints (solvency cone of financial positions with non negative liquidation values) and consumption constraints, respectively. The dynamics of a portfolio process is defined by:

$$
d V_{t}^{i}=V_{t-}^{i} d Y_{t}^{i}+d B_{t}^{i}-d C_{t}^{i}, \quad i=1, \ldots, d, \quad V_{0}=V_{0-}=x,
$$

where the controls $\pi=(B, C)$ are làdlàg predictable processes of finite variations. The dynamics (2.3) means that the portfolio process $V$ is self-financing. The variations of $V$ are only due to the increments of $Y$. The transaction costs described by $B$ are withdrawn from the portfolio value. At last, $C$ represents the cumulated sum of consumed 
wealth. If $x \in \mathbf{R}^{d}$ is an initial endowment, we assume that $\pi=(B, C)$ belongs to the class denoted by $\mathcal{A}_{x}$ of all admissible controls satisfying the following properties:

1) $\dot{B}^{c} \in-K, \mathrm{~d} \mathbb{P}, \mathrm{d}\left\|B^{c}\right\|$ a.e., $\quad \dot{C}^{c} \in \mathcal{C}, \mathrm{d} \mathbb{P}, \mathrm{d}\left\|C^{c}\right\|$ a.e.,

2) $\Delta^{+} B_{\tau} \in-K, \Delta^{+} C_{\tau} \in \mathcal{C}$, a.s. for all stopping times $\tau$,

3) $\Delta B_{\tau} \in-K, \Delta C_{\tau} \in \mathcal{C}$, a.s. for all predictable stopping times $\tau$,

4) If $V=V^{\pi}, \pi=(B, C) \in \mathcal{A}_{x}$, is such that $V_{\tau} \in \operatorname{int} K$ for some stopping time $\tau$, then $V_{\tau}^{+}=V_{\tau}+\Delta^{+} B_{\tau}-\Delta^{+} C_{\tau} \in \operatorname{int} K$,

5) If $V=V^{\pi}, \pi=(B, C) \in \mathcal{A}_{x}$, is such that $V_{\tau-} \in \operatorname{int} K$ (resp. $K$ ) for some stopping time $\tau$, then $V_{\tau-}+\Delta B_{\tau}-\Delta C_{\tau} \in \operatorname{int} K($ resp. $K)$,

6) $x+\Delta^{+} B_{0} \in \operatorname{int} K$.

The three last conditions mean that portfolio manager does not deliberately get his position out of the solvency cone. It is also assumed that $\Delta B_{0}^{+}=\Delta C_{0}=\Delta^{+} C_{0}=0$ and $d C^{c}$ is absolutely continuous with respect to the Lebesgue measure and we write $d C_{t}^{c}=c_{t} d t$.

Using the monotonicity of the controls $B, C$ with respect to the partial order induced by $K$ (i.e. $\forall x, y \in \mathbf{R}^{d}, x \preceq y \Leftrightarrow y-x \in K$ ), we may deduce that $B$ and $C$ are of finite variations. Indeed, since $\operatorname{int} K \neq \emptyset$, by an appropriate change of coordinates we may assume w.l.o.g. that all coordinates of $B, C$ are monotonic, hence are of finite variations.

Without loss of generality, we assume that $C=C^{c}$ is continuous as the jumps of $C$ are ignored in the optimization problem we consider. Precisely, we assume that $d C_{t}=c_{t} d t$ almost everywhere w.r.t. the Lesbegue $d t$ measure on $\mathbf{R}$.

For every control $\pi \in \mathcal{A}_{x}$, let us introduce the stopping time

$$
\theta^{\pi}=\inf \left\{t: V_{t}^{\pi} \notin \operatorname{int} K\right\}
$$

where $V^{\pi}$ is the portfolio process starting from $x \in \mathbf{R}^{d}$ and satisfying (2.3). We suppose that the strategy $\pi=(B, C)$ is frozen after the exit time, i.e. $\Delta^{+} B_{\theta}=0$ and $d B_{t}=$ $c_{t}=0$ for $t>\theta$. Throughout the paper, we fix a discount coefficient $\beta>0$.

For every control $\pi=(B, C) \in \mathcal{A}_{x}, x \in \operatorname{int} K$, we define the utility process

$$
J_{t}^{\pi}(x):=\int_{0}^{t \wedge \theta^{\pi}} e^{-\beta s} U\left(c_{s}\right) d s,
$$

where $U$ is a non-negative utility function defined on $\mathcal{C}$. We assume that $U$ is concave, $U(0)=0$ and $U(x) /|x| \rightarrow 0$ as $|x| \rightarrow \infty$. The optimal consumption problem consists in optimizing the utility process $J^{\pi}(x)$ over the set of all admissible strategies. To do so, we define the Bellman function as

$$
W(x):=\sup _{\pi \in \mathcal{A}_{x}} \mathbb{E} J_{\infty}^{\pi}(x), \quad x \in \operatorname{int} K
$$

Showing that the Bellman function is finite is not a trivial task. Indeed, this is based on the existence of classical supersolutions to the associated HJB equation, see Lemma 6.2. Moreover, the continuity of $W$ is proven in Theorem 7.4 in Appendix. 


\section{Some elementary properties of the Bellman function}

We denote by $\succeq$ the partial order defined by $K$, i.e. if $x, y \in \mathbf{R}^{d}, x \succeq y \Leftrightarrow x-y \in K$.

Proposition 3.1 The function $W$ is increasing with respect to the partial order $\succeq$.

Proof. Suppose that $x_{2} \succeq x_{1}$. Let $\pi=(B, C) \in \mathcal{A}_{x_{1}}$ and $V^{(1)}$ be such that

$$
\begin{aligned}
V_{0-}^{(1)} & =x_{1}, \\
d V_{t}^{(1) i} & =V_{t_{-}}^{(1) i} d Y_{t}^{i}+d B_{t}^{i}-d C_{t}^{i}, \quad t \geq 0, i=1, \cdots, d .
\end{aligned}
$$

Let us define $V_{0-}^{(2)}=x_{2}$ and $V_{t}^{(2)}=V_{t}^{(1)}$ if $t \geq 0$. From the dynamics of $V^{(1)}$ we deduce that

$$
\begin{aligned}
V_{0-}^{(2)} & =x_{2}, \\
d V_{t}^{(2) i} & =V_{t_{-}}^{(2) i} d Y_{t}^{i}+d \tilde{B}_{t}^{i}-d C_{t}^{i}, \quad t \geq 0, i=1, \cdots, d,
\end{aligned}
$$

where $\tilde{B}_{t}^{i}=B_{t}^{i}+\left(x_{1}^{i}-x_{2}^{i}\right) I_{[0, \infty)}(t)$ is still a làdlàg and predictable process of finite variations satisfying $\Delta \tilde{B}_{t} \in-K$ a.s. since $x_{1}-x_{2} \in-K$. As $V_{0-}^{(2)}, V_{0-}^{(1)} \in \operatorname{int} K$ it is straightforward that $\theta^{2}=\theta^{1}$ where $\theta^{i}, i=1,2$, are the stopping times defined by (2.4) respectively for $V^{(2)}$ and $V^{(1)}$. It follows that $\widetilde{\pi}=(\widetilde{B}, C) \in \mathcal{A}_{x_{2}}$ and we deduce that $W\left(x_{1}\right) \leq W\left(x_{2}\right)$.

In the following, we obtain lower bounds for the Bellman function. To do so, let us define the liquidation function associated to the solvency cone $K$, i.e. for $x \in K$,

$$
l(x):=\sup \left\{z \in \mathbf{R}_{+}: x-z e_{1} \in K\right\} .
$$

We have $x-l(x) e_{1} \in K$. Then, consider the strategy $\Delta B_{0}:=l(x) e_{1}-x$ and $B_{t}=B_{0}$ for $t \geq 0$. For a given consumption plan $c$, the corresponding portfolio process is: $V_{t}=\left(X_{t}, 0\right)$ where

$$
X_{t}:=l(x) S_{t}^{1}-S_{t}^{1} \int_{0}^{t}\left(S_{u}^{1}\right)^{-1} c_{u} d u .
$$

If the consumption plan is $c_{s}=\kappa X_{s}$, then $Y_{t}:=X_{t}\left(S_{t}^{1}\right)^{-1}$ satisfies

$$
Y_{t}:=l(x)-\kappa \int_{0}^{t} Y_{u} d u
$$

i.e. $Y_{t}=l(x) e^{-\kappa t}$. It follows that $X_{t}=S_{t}^{1} l(x) e^{-\kappa t}$. We deduce that the process $X$ does not hit zero, i.e. the process $V$ stays in the interior of the solvency cone. With the strategy $\pi:=(B, C)$ above,

$$
J_{\infty}^{\pi}=\int_{0}^{\infty} e^{-\beta t} U\left(\kappa S_{t}^{1} l(x) e^{-\kappa t}\right) d t
$$

and then,

$$
W(x) \geq \sup _{\kappa>0} E \int_{0}^{\infty} e^{-\beta t} U\left(\kappa S_{t}^{1} l(x) e^{-\kappa t}\right) d t .
$$

In the particular case where $Y_{t}^{1}=0$, i.e. $S_{t}^{1}=1$, we have the following: 
Lemma 3.2 With $S^{1} \equiv 1$ and the power utility function $U(x)=u_{\gamma}\left(x e_{0}\right)$, where $u_{\gamma}(t)=t^{\gamma} / \gamma, \gamma \in(0,1)$, we have

$$
W(x) \geq \frac{1}{\gamma} \kappa_{*}^{\gamma-1} l^{\gamma}(x)=\frac{1}{\gamma}\left(\frac{\beta}{1-\gamma}\right)^{\gamma-1} l^{\gamma}(x),
$$

where $\kappa_{*}:=\beta /(1-\gamma)$.

\section{The HJB equation}

In the following, we denote by $C_{p}(K)$ the set of all continuous functions $f$ on $K$ such that $\sup _{x \in K}|f(x)|(1+|x|)^{-p}<\infty$. The set of all functions $f$ which are $C^{2}$, i.e. twice continuously differentiable on int $K$ is denoted by $C^{2}(K)$. We use the notation $C^{2}(x)$, $x \in \mathbf{R}^{2}$, for the functions having only these properties on a neighbourhood of $x$. For each $\pi=(B, C) \in \mathcal{A}_{x}, x \in \operatorname{int} K$, and every function $f \in C_{1}(K) \cap C^{2}(x)$ which are increasing with respect to the order $\preceq_{K}$, we consider the integro-differential operator

$$
H(f, x):=\int_{\mathbf{R}^{d}}\left[f(x+\operatorname{diag}(x) z) I(x, z)-f(x)-f^{\prime}(x) \operatorname{diag}(x) z\right] \Pi(d z),
$$

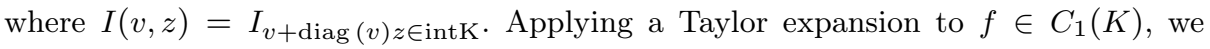
claim that

$$
\left|f(x+\operatorname{diag}(x) z) I(x, z)-f(x)-f^{\prime}(x) \operatorname{diag}(x) z\right| \leq C_{x}\left(|z| \wedge|z|^{2}\right),
$$

where $C_{x}$ is a constant depending on $x$. Therefore, the operator $H$ is well defined. Let us now define

$$
\begin{aligned}
G: & =(-K) \cap \partial \mathcal{O}_{1}(0), \\
\Sigma_{G}(p) & :=\sup _{x \in G} p x \\
U^{*}(p) & :=\sup _{x \in \mathcal{C}}(U(x)-p x), \\
A v: & =(\operatorname{diag} \mathrm{v}) . \Xi((\operatorname{diag} \mathrm{v}) . \Xi)^{T}, \quad v \in \mathbf{R}^{d},
\end{aligned}
$$

and the operators:

$$
\begin{aligned}
F_{0}(X, p, H, W, x) & :=\frac{1}{2} \operatorname{Tr} A(x) X+\mu^{T}(\operatorname{diag} x) p+H-\beta W, \\
\mathcal{L}_{0} \phi & :=F_{0}\left(\phi^{\prime \prime}(x), \phi^{\prime}(x), H(\phi, x), \phi(x), x\right) \\
F(X, p, H, W, x): & =\max \left\{F_{0}(X, p, H, W, x)+U^{*}(p), \Sigma_{G}(p)\right\}, \\
\mathcal{L} \phi & :=F\left(\phi^{\prime \prime}(x), \phi^{\prime}(x), H(\phi, x), \phi(x), x\right) .
\end{aligned}
$$

Let us introduce the Dirichlet problem associated to the HJB equation:

$$
\begin{aligned}
& F\left(W^{\prime \prime}(x), W^{\prime}(x), H(W, x), W(x), x\right)=0, \quad x \in \operatorname{int} K, \\
& W(x)=0, \quad \text { on } \partial K .
\end{aligned}
$$


We consider a possible solution to the HJB equation in the viscosity sense. Observe that the integro-differential operator $H$ is not locally defined because of the operator $H$. Therefore, we define viscosity solutions in the global sense as follows:

Definition 4.1 A function $v \in C(K)$ is called a viscosity supersolution of (4.12) on a subset $\tilde{K} \subseteq K$ if for every $x \in \operatorname{int} \tilde{K}$ and every $f \in C_{1}(K) \cap C^{2}(x)$ such that $v(x)=f(x)$ and $v \geq f$ on $K$, the inequality $\mathcal{L} f(x) \leq 0$ holds.

Definition 4.2 A function $v \in C(K)$ is called a viscosity subsolution of (4.12) on a subset $\tilde{K} \subseteq K$ if for every $x \in \operatorname{int} \tilde{K}$ and every $f \in C_{1}(K) \cap C^{2}(x)$ such that $v(x)=f(x)$ and $v \leq f$ on $K$, the inequality $\mathcal{L} f(x) \geq 0$ holds.

Definition 4.3 A function $v \in C(K)$ is called a viscosity solution of (4.12) on a subset $\tilde{K} \subseteq K$ if $v$ is simultaneously a viscosity super- and subsolution on the subset $\tilde{K} \subseteq K$.

When, the subset $\tilde{K}=K$, we just say that $v$ is a viscosity solution (resp. super- or subsolution). At last, a function $v \in C_{1}(K) \cap C^{2}$ (int $K$ ) is called classical supersolution of (4.12) on a subset $\tilde{K} \subseteq K$ if $\mathcal{L} v \leq 0$ on int $\tilde{K}$. We add the adjective strict when $\mathcal{L} v<0$ on the set int $K$.

Lemma 4.4 A function $v \in C(K)$ is a viscosity supersolution of (4.12) on a subset $\tilde{K} \subseteq K$ if and only if, for every point $x \in \operatorname{int} \tilde{K}$, the inequality $\mathcal{L} f(x) \leq 0$ holds for any function $\phi \in C^{2}(x)$, such that the difference $v-\phi$ attains its global minimum on $K$ at $x$.

Proof. The proof of this result is the same than the proof of [9, Lemma 4.2.4] except that we replace the notion of local minimum by the global one.

Remark 4.5 In the classical theory developed for differential equations, the notion of viscosity solution admits an equivalent formulation in terms of super-and subjets $\mathrm{J}^{+}$, $J^{-}$(see definition in [4]). But this is not the case in our formulation due to the nonlocal property of the integro-differential operator. Although, there is a link between the notion of viscosity solutions and super- and subjets as stated in [4, Section 7].

Theorem 4.6 Suppose that $W$ takes finite values. Then,

i) The Bellman function $W$ is a viscosity supersolution to (4.12).

ii) The Bellman function $W$ is a viscosity subsolution to (4.12).

Theorem 4.6 implies that the Bellman function is a viscosity solution to (4.12). We shall see conditions under which $W$ is finite.

\subsection{Proof of Theorem 4.6}

The proof is based on preliminary results we formulate before. The proof of the following lemma is given in [4].

Lemma 4.7 For every portfolio process $V=V^{\pi}, \pi \in \mathcal{A}_{x}$, the process $I_{V_{-} \in \partial K}|\Delta Y| I_{\left[0, \theta^{\pi}\right]}$ is indistinguishable from zero. 
Let us define for each $n$ the compact set

$$
K_{n}:=\{x \in K:|x| \leq n\} \cap\left\{x \in K: d(x, \partial K) \geq \frac{1}{n}\right\} .
$$

Note that $\left(K_{n}\right)_{n \geq 1}$ is an increasing sequence whose union is int $K$. For every control $\pi=(B, C) \in \mathcal{A}_{x}$ and $x \in \operatorname{int} K$, we define $V^{\theta_{+}^{n}}$ as the stopped portfolio process.

$$
V^{\theta_{+}^{n}}=V^{\pi, x, \theta_{+}^{n}}:=V^{\pi, x} I_{\left[0, \theta^{n}[\right.}+V_{\theta_{+}^{n}}^{\pi, x} I_{\left[\theta^{n}, \infty[,\right.}
$$

where $\theta^{n}$ is the first instant when the portfolio exits $K_{n}$. We also define $B^{\theta^{n}}$ similarly. Note that the value of $V_{\theta^{n}}^{\pi, x}$ may be outside int $K$ due to a possible jump of the Lévy process at $\theta^{n}$ but, in this case, $V_{\theta^{n}}^{\pi, x} \notin \partial K$ by virtue of Lemma 4.7. From the dynamics of $V^{\pi, x}$, we deduce that

$$
\begin{aligned}
V_{t}^{\theta_{+}^{n}} & =x+\int_{0}^{t} I_{\left[0, \theta^{n}\right]}(s) \operatorname{diag}\left(V_{s-}^{\theta_{+}^{n}}\right)\left(\mu_{s} d s+\Xi d W_{s}\right)+B_{t}^{\theta_{+}^{n}}-C_{t}^{\theta^{n}}, \\
& +\int_{0}^{t} \int_{\mathbf{R}^{d}} \operatorname{diag}\left(V_{s-}^{\theta_{+}^{n}}\right) z I_{\left[0, \theta^{n}\right]}(s)(p(d z, d s)-q(d z, d s)) .
\end{aligned}
$$

We propose to study the quantity

$$
\bar{X}_{t}^{f, n}:=e^{-\beta t} f\left(V_{t+}^{\theta_{+}^{n}}\right) I\left(V_{t-}^{\theta_{+}^{n}}, \Delta Y_{t \wedge \theta_{n}}\right)+J_{t}^{\pi},
$$

by means of the Ito formula. We set $f\left(V_{t+}^{\theta_{+}^{n}}\right) I\left(V_{t-}^{\theta_{+}^{n}}, \Delta Y_{t \wedge \theta_{n}}\right)=0$ by convention when $I\left(V_{t-}^{\theta_{+}^{n}}, \Delta Y_{t \wedge \theta_{n}}\right)=0$ so that $\bar{X}_{t}^{f, n}$ is well defined even if $f$ is only defined on $K$. If $K_{n}$ is replaced by $K$ we write $\bar{X}_{t}^{f}$ instead of $\bar{X}_{t}^{f, n}$. We also introduce

$$
\widetilde{V}_{t}^{n}:=V_{t+} I_{\left[0, \theta^{n}[\right.}(t)+V_{\theta_{-}^{n}} I_{\left[\theta^{n}, \infty[\right.}(t) .
$$

We have the following key result:

Lemma 4.8 (Itô expansion) Let $f \in C_{1}(K) \cap C^{2}$ (int $\left.\mathrm{K}\right)$ be an increasing function with respect to the order $\preceq_{K}$. Then, we have

$$
\begin{aligned}
\bar{X}_{t}^{f, n} & =f(x)+N_{t \wedge \theta^{n}}+R_{t \wedge \theta^{n}} \\
& +\int_{0}^{t \wedge \theta^{n}} e^{-\beta u}\left[\mathcal{L}_{0} f\left(\widetilde{V}_{u_{-}}^{n}\right)-f^{\prime}\left(\widetilde{V}_{u_{-}}^{n}\right) c_{u}+U\left(c_{u}\right)\right] d u,
\end{aligned}
$$

where $N$ is a local martingale and $R$ is a decreasing process such that $R_{0}=0$.

Proof. Note that we do not assume any regularity of $f$ on $\partial K$. Therefore, we can not directly apply the Ito formula to $\bar{X}_{t}^{f, n}$. To overcome this difficulty, instead of considering $V^{\theta_{+}^{n}}$, we study the process $V^{\theta_{-}^{n}}$ defined by

$$
V^{\theta_{-}^{n}}:=V I_{\left[0, \theta^{n}[\right.}+V_{\theta_{-}^{n}} I_{\left[\theta^{n}, \infty[\right.} \cdot
$$

We also have a representation for $V^{\theta_{-}^{n}}$ :

$$
\begin{aligned}
V_{t}^{\theta_{-}^{n}} & =x+\int_{0}^{t} I_{\left[0, \theta^{n}\right]}(s) \operatorname{diag}\left(V_{s-}^{\theta_{-}^{n}}\right)\left(\mu_{s} d s+\Xi d W_{s}\right)+B_{t}^{\theta_{-}^{n}}-C_{t}^{\theta^{n}} \\
& +\int_{0}^{t} \int_{\mathbf{R}^{d}} \operatorname{diag}\left(V_{s-}^{\theta_{-}^{n}}\right) z I_{\left[0, \theta^{n}\right)}(s)(p(d z, d s)-q(d z, d s)) .
\end{aligned}
$$


For a sake of simplicity, we write $\widetilde{V}_{t}:=V_{t+}^{\theta_{-}^{n}}=V_{t}^{\theta_{-}^{n}}+\Delta^{+} B_{t}^{\theta_{-}^{n}}$. The Itô formula applied to the process $e^{-\beta t} f\left(\widetilde{V}_{t}\right):=e^{-\beta t} f\left(V_{t+}^{\theta_{-}^{n}}\right)$ yields

$$
\begin{aligned}
e^{-\beta t} f\left(\widetilde{V}_{t}\right) & =f(x)+\int_{0}^{t} e^{-\beta u} f^{\prime}\left(\widetilde{V}_{u_{-}}\right) d \widetilde{V}_{u}-\beta \int_{0}^{t} e^{-\beta u} f\left(\widetilde{V}_{u_{-}}\right) I_{\left[0, \theta^{n}\right]}(s) d u \\
& +\frac{1}{2} \int_{0}^{t} e^{-\beta s} \operatorname{Tr} A \widetilde{V}_{s_{-}} f^{\prime \prime}\left(\widetilde{V}_{s_{-}}\right) I_{\left[0, \theta^{n}\right]}(s) d s \\
& +\sum_{s \leq t} e^{-\beta s}\left[f\left(\widetilde{V}_{s}\right)-f\left(\widetilde{V}_{s_{-}}\right)-f^{\prime}\left(\widetilde{V}_{s_{-}}\right)\left(\widetilde{V}_{s}-\widetilde{V}_{s_{-}}\right)\right]
\end{aligned}
$$

Using (4.16) and the fact that $B_{t}^{\theta_{-}^{n}}+\Delta^{+} B_{t}^{\theta_{-}^{n}}=B_{t+} 1_{\left[0, \theta^{n}\right)}+B_{\theta^{n}-} 1_{\left[\theta^{n}, \infty\right)}$, we deduce that

$$
\begin{aligned}
\int_{0}^{t} e^{-\beta u} f^{\prime}\left(\widetilde{V}_{u_{-}}\right) d \widetilde{V}_{u} & =\int_{0}^{t} e^{-\beta u} f^{\prime}\left(\widetilde{V}_{u_{-}}\right) I_{\left[0, \theta^{n}\right)}(u) \operatorname{diag}\left(\widetilde{V}_{u-}\right)\left(\mu_{u} d u+\Xi d W_{u}\right) \\
& +\int_{0}^{t} e^{-\beta u} f^{\prime}\left(\widetilde{V}_{u_{-}}\right) d B_{u}^{c}+\int_{0}^{t} e^{-\beta u} f^{\prime}\left(\widetilde{V}_{u_{-}}\right) c_{u} d u \\
& +\sum_{s \leq t} e^{-\beta s} I_{\left[0, \theta^{n}\right)}(s) f^{\prime}\left(\widetilde{V}_{s_{-}}\right)\left(\Delta^{+} B_{s}+\Delta B_{s}\right) \\
& +\int_{0}^{t} \int_{\mathbf{R}^{d}} e^{-\beta u} f^{\prime}\left(\widetilde{V}_{u_{-}}\right) \operatorname{diag}\left(\widetilde{V}_{s_{-}}\right) z I_{\left[0, \theta^{n}\right)}(s)(p(d z, d s)-q(d z, d s)) .
\end{aligned}
$$

Note that $\widetilde{V}_{s}=\widetilde{V}_{s_{-}}+\operatorname{diag} \widetilde{V}_{s_{-}} \Delta Y_{s}+\Delta^{+} B_{s}+\Delta B_{s}$. We then rewrite

$$
\begin{aligned}
& \sum_{s \leq t} e^{-\beta s}\left[f\left(\widetilde{V}_{s}\right)-f\left(\widetilde{V}_{s_{-}}\right)-f^{\prime}\left(\widetilde{V}_{s_{-}}\right)\left(\widetilde{V}_{s}-\widetilde{V}_{s_{-}}\right)\right] \\
= & \sum_{s \leq t} e^{-\beta s}\left[f\left(\widetilde{V}_{s}\right)-f\left(\widetilde{V}_{s_{-}}+\operatorname{diag} \widetilde{V}_{s_{-}} \Delta Y_{s}\right)\right] I_{\left[0, \theta^{n}\right)}(s) \\
+ & \sum_{s \leq t} e^{-\beta s}\left[f\left(\widetilde{V}_{s_{-}}+\operatorname{diag}\left(\widetilde{V}_{s_{-}}\right) \Delta Y_{s}\right)-f\left(\widetilde{V}_{s_{-}}\right)-f^{\prime}\left(\widetilde{V}_{s_{-}}\right) \operatorname{diag}\left(\widetilde{V}_{s_{-}}\right) \Delta Y_{s}\right] I_{\left[0, \theta^{n}\right)}(s) \\
- & \sum_{s \leq t} e^{-\beta s} f^{\prime}\left(\widetilde{V}_{s_{-}}\right)\left(\Delta^{+} B_{s}+\Delta B_{s}\right) I_{\left[0, \theta^{n}\right)}(s) .
\end{aligned}
$$

Moreover,

$$
\begin{aligned}
& \sum_{s \leq t} e^{-\beta s}\left[f\left(\widetilde{V}_{s_{-}}+\operatorname{diag}\left(\widetilde{V}_{s_{-}}\right) \Delta Y_{s}\right)-f\left(\widetilde{V}_{s_{-}}\right)-f^{\prime}\left(\widetilde{V}_{s_{-}}\right) \operatorname{diag}\left(\widetilde{V}_{s_{-}}\right) \Delta Y_{s}\right] I_{\left[0, \theta^{n}\right)}(s) \\
= & \int_{0}^{t} \int_{\mathbf{R}^{d}} e^{-\beta s}\left[f\left(\widetilde{V}_{s_{-}}+\operatorname{diag}\left(\widetilde{V}_{s_{-}}\right) z\right)-f\left(\widetilde{V}_{s_{-}}\right)-f^{\prime}\left(\widetilde{V}_{s_{-}}\right) \operatorname{diag}\left(\widetilde{V}_{s_{-}}\right) z\right] I_{\left[0, \theta^{n}\right)}(s) p(d z, d s) .
\end{aligned}
$$

Since $I\left(\widetilde{V}_{s_{-}}, \Delta Y_{s}\right)=1$ for $s<\theta^{n}$, we may omit the indicator $I$ within the operator $H$ for $s<\theta^{n}$. We deduce that

$$
\begin{aligned}
e^{-\beta t} f\left(\widetilde{V}_{t}\right) & =f(x)+\tilde{N}_{t \wedge \theta^{n}}+\tilde{R}_{t \wedge \theta^{n}} \\
& +\int_{0}^{t \wedge \theta^{n}} e^{-\beta u}\left[\mathcal{L}_{0} f\left(\widetilde{V}_{u_{-}}\right)-f^{\prime}\left(\widetilde{V}_{u_{-}}\right) c_{u}\right] d u
\end{aligned}
$$


where

$$
\begin{aligned}
\tilde{N}_{t} & =\int_{0}^{t} e^{-\beta u} f^{\prime}\left(\widetilde{V}_{u_{-}}\right) \operatorname{diag}\left(\widetilde{V}_{u-}\right) \Xi I_{\left[0, \theta^{n}\right]}(u) d W_{u} \\
& +\int_{0}^{t} \int_{\mathbf{R}^{d}} e^{-\beta u}\left[f\left(\widetilde{V}_{u_{-}}+\operatorname{diag}\left(\widetilde{V}_{u_{-}}\right) z\right)-f\left(\widetilde{V}_{u_{-}}\right)\right] I_{\left[0, \theta^{n}\right)}(u)(p(d z, d u)-q(d z, d u)) .
\end{aligned}
$$

The residual term is

$$
\begin{aligned}
\tilde{R}_{t}: & =\sum_{s \leq t} e^{-\beta s} I_{\left[0, \theta^{n}\right)}(s)\left[f\left(\widetilde{V}_{s_{-}}+\operatorname{diag}\left(\widetilde{V}_{s_{-}}\right) \Delta Y_{s}+\Delta B_{s}^{+}+\Delta B_{s}\right)\right. \\
& \left.-f\left(\widetilde{V}_{s_{-}}+\operatorname{diag}\left(\widetilde{V}_{s_{-}}\right) \Delta Y_{s}\right)\right]+\int_{0}^{t} e^{-\beta u} f^{\prime}\left(\widetilde{V}_{u_{-}}\right) I_{\left[0, \theta^{n}\right)}(u) d B_{u}^{c} .
\end{aligned}
$$

The process $\tilde{R}$ is decreasing due to the monotonicity of $f$ with respect to $K$. Finally, observe that

$$
\begin{aligned}
\bar{X}_{t}^{f, n}-e^{-\beta t} f\left(\widetilde{V}_{t}\right) & =J_{t}^{\pi}+e^{-\beta \theta^{n}}\left[f\left(V_{\theta^{n}+}\right) I\left(V_{\theta^{n}-}, \Delta Y_{\theta_{n}}\right)-f\left(V_{\theta^{n}-}\right)\right] 1_{t=\theta^{n}}, \\
& =J_{t}^{\pi}+e^{-\beta \theta^{n}}\left[f\left(V_{\theta^{n}+}\right)-f\left(V_{\theta^{n}-}+\operatorname{diag}\left(V_{\theta_{-}^{n}}\right) \Delta Y_{\theta^{n}}\right)\right] I\left(V_{\theta^{n}-}, \Delta Y_{\theta_{n}}\right) 1_{t=\theta^{n}} \\
& +e^{-\beta \theta^{n}}\left[f\left(V_{\theta^{n}-}+\operatorname{diag}\left(V_{\theta_{-}^{n}}\right) \Delta Y_{\theta^{n}}\right) I\left(V_{\theta^{n}-}, \Delta Y_{\theta_{n}}\right)-f\left(V_{\theta^{n}-}\right)\right] 1_{t=\theta^{n}} .
\end{aligned}
$$

Plugging the r.h.s of (4.21) into (4.17), as the Lesbesgue measure $d t$ does not charge any point, we conclude the theorem with

$$
\begin{aligned}
\tilde{N}_{t} & =\int_{0}^{t} e^{-\beta u} f^{\prime}\left(V_{u_{-}}\right) \operatorname{diag}\left(V_{u-}\right) \Xi I_{\left[0, \theta^{n}\right]}(u) d W_{u} \\
& +\int_{0}^{t} \int_{\mathbf{R}^{d}} e^{-\beta u}\left[f\left(V_{u_{-}}+\operatorname{diag}\left(V_{u_{-}}\right) z\right) I\left(V_{u_{-}}, \Delta Y_{u}\right)-f\left(V_{u_{-}}\right)\right] I_{\left[0, \theta^{n}\right]}(u)(p(d z, d u)-q(d z, d u)),
\end{aligned}
$$

and

$$
\begin{aligned}
\tilde{R}_{t}: & =\sum_{s \leq t} e^{-\beta s} I_{\left[0, \theta^{n}\right]}(s)\left[f\left(V_{s_{-}}+\operatorname{diag}\left(V_{s_{-}}\right) \Delta Y_{s}+\Delta^{+} B_{s}+\Delta B_{s}\right)\right. \\
& \left.-f\left(V_{s_{-}}+\operatorname{diag}\left(V_{s_{-}}\right) \Delta Y_{s}\right)\right] I\left(V_{s_{-}}, \Delta Y_{s}\right)+\int_{0}^{t} e^{-\beta u} f^{\prime}\left(V_{u-}\right) I_{\left[0, \theta^{n}\right]}(s) d B_{s}^{c} .
\end{aligned}
$$

Remark 4.9 If the function $f \in C^{2}\left(\mathbf{R}^{d}\right)$, we may directly apply the Ito formula to $X_{t}^{f}:=e^{-\beta t} f\left(V_{t+}\right)+J_{t}^{\pi}$ and we obtain the following result:

$$
X_{t}^{f}=f(x)+N_{t}+R_{t}+\int_{0}^{t \wedge \theta} e^{-\beta u}\left[\mathcal{L}_{0} f\left(V_{u_{-}}\right)-f^{\prime}\left(V_{u_{-}}\right) c_{u}+U\left(c_{u}\right)\right] d u,
$$

where $N$ is a local martingale and $R$ is a decreasing process with $R_{0}=0$. Moreover, $X_{t}^{f}=\bar{X}_{t}^{f}$ if $f$ vanishes outside int $K$. 
We now formulate a strict supersolution property which is the key point to deduce that $\mathrm{W}$ is a global viscosity subsolution of the HJB equation by using the dynamic programming principle. We fix a closed ball such that $\overline{\mathcal{O}_{r}}(x) \subseteq \mathcal{O}_{2 r}(x) \subseteq \operatorname{int} K$ and we define the stopping time $\tau^{\pi}:=\tau_{r}^{\pi}$ as the exit time of $V^{\pi, x}$ from $\mathcal{O}_{r}(x)$, i.e.

$$
\tau^{\pi}:=\inf \left\{t \geq 0:\left|V_{t}^{\pi, x}-x\right| \geq r\right\} .
$$

Lemma 4.10 Let $f \in C^{2}(K) \cap C_{1}(K)$ be such that $\mathcal{L} f \leq-\varepsilon \leq 0$ on $\overline{\mathcal{O}_{r}}(x)$. There exists a constant $\eta:=\eta(\varepsilon, r)$ and an interval $\left(0, t_{0}\right]$ such that

$$
\sup _{\pi \in \mathcal{A}_{x}} E \bar{X}_{t \wedge \tau^{\pi}}^{f, n} \leq f(x)-\eta t, \quad t \in\left(0, t_{0}\right]
$$

where $t \mapsto \bar{X}_{t \wedge \tau^{\pi}}^{f, n}$ is defined by (4.15).

Proof. We fix a strategy $\pi$ and omit its symbol in the notations below. Observe that only the behaviour of the processes we consider does matter on $[0, \tau]$. For $n$ large enough, we have $\mathcal{O}_{2 r}(x) \subseteq \operatorname{int} K_{n}$. Therefore, $\tau^{\pi} \leq \theta^{n}$ hence we may apply Lemma 4.8 so that

$$
\begin{aligned}
\bar{X}_{t \wedge \tau}^{f, n} & =f(x)+N_{t \wedge \tau}+R_{t \wedge \tau}+\int_{0}^{t \wedge \tau} e^{-\beta u}\left[\mathcal{L}_{0} f\left(V_{u_{-}}\right)+U^{*}\left(V_{u-}\right)\right] d u \\
& -\int_{0}^{t \wedge \tau} e^{-\beta u}\left[U^{*}\left(V_{u-}\right)+f^{\prime}\left(V_{u_{-}}\right) c_{u}-U\left(c_{u}\right)\right] d u \\
& =f(x)+N_{t \wedge \tau}+R_{t \wedge \tau}+\int_{0}^{t \wedge \tau} e^{-\beta u} \mathcal{L} f\left(V_{u_{-}}\right) d u \\
& -\int_{0}^{t \wedge \tau} e^{-\beta u}\left[U^{*}\left(V_{u-}\right)+f^{\prime}\left(V_{u_{-}}\right) c_{u}-U\left(c_{u}\right)\right] d u \\
& +\int_{0}^{t \wedge \tau} e^{-\beta u}\left[\mathcal{L}_{0} f\left(V_{u_{-}}\right)+U^{*}\left(V_{u-}\right)-\mathcal{L} f\left(V_{u_{-}}\right)\right] d u
\end{aligned}
$$

We deduce that

$$
\begin{aligned}
\bar{X}_{t \wedge \tau}^{f, n} & =f(x)+N_{t \wedge \tau}+\widetilde{R}_{t \wedge \tau}+\int_{0}^{t \wedge \tau} e^{-\beta u} \mathcal{L} f\left(V_{u_{-}}\right) d u \\
& -\int_{0}^{t \wedge \tau} e^{-\beta u}\left[U^{*}\left(V_{u-}\right)+f^{\prime}\left(V_{u_{-}}\right) c_{u}-U\left(c_{u}\right)\right] d u
\end{aligned}
$$

where $\widetilde{R}$ is a decreasing process such that $\widetilde{R}_{0}=0$ and $N$ is a local martingale. As it is shown in Lemma 8.3 [4], the stopped process $N^{\tau}$ is a martingale hence $\mathbb{E} N^{t \wedge \tau}=0$.

By assumption, $\mathcal{L} f(y) \leq-\varepsilon$ for all $y \in \overline{\mathcal{O}_{r}}(x)$ and so $\Sigma_{G}\left(f^{\prime}(y)\right) \leq-\varepsilon$ on $[0, \tau]$. It follows that $f^{\prime}(y) k \leq-\varepsilon|k|$ whatever $k \in-K$ so that $f^{\prime}\left(\overline{\mathcal{O}_{r}}(x)\right) \subseteq \operatorname{int} K^{*}$ on $[0, \tau]$. In particular, for $s \in[0, \tau], f^{\prime}\left(V_{s_{-}}\right) \dot{B}_{s}^{c} \leq-\varepsilon\left|\dot{B}_{s}^{c}\right|$. We deduce that the following term above (appearing in the expression (4.23) of $R$ ) is bounded as follows:

$$
\int_{0}^{t \wedge \tau} e^{-\beta u} f^{\prime}\left(V_{u_{-}}\right) I_{u \leq \theta} \dot{B}_{u}^{c} d\left\|B^{c}\right\|_{u} \leq-\varepsilon \int_{0}^{t \wedge \tau} e^{-\beta u} I_{u \leq \theta}\left|\dot{B}_{u}^{c}\right| d\left\|B^{c}\right\|_{u}
$$

On the other hand, the other terms defining $R$ in (4.23) can be estimated as follows: 


$$
\begin{aligned}
& f\left(V_{s_{-}}+\operatorname{diag}\left(V_{s_{-}}\right) \Delta Y_{s}+\Delta B_{s}^{+}+\Delta B_{s}\right)-f\left(V_{s_{-}}+\operatorname{diag}\left(V_{s_{-}}\right) \Delta Y_{s}\right) \\
= & \left(f\left(V_{s_{+}}\right)-f\left(V_{s}\right)\right) 1_{\Delta B_{s}=0}+\left(f\left(V_{s_{+}}\right)-f\left(V_{s-}\right)\right) 1_{\Delta B_{s} \neq 0,}, \\
= & f^{\prime}\left(\gamma_{s}\right)\left(\Delta B_{s}+\Delta^{+} B_{s}\right),
\end{aligned}
$$

where $\gamma_{s} \in\left[V_{s}, V_{s+}\right]$ on the set $\left\{\Delta B_{s}=0\right\}$ and $\gamma_{s} \in\left[V_{s-}, V_{s+}\right]$ on the set $\left\{\Delta B_{s} \neq 0\right\}$. Observe that $\gamma_{s} \in \overline{\mathcal{O}_{r}}(x)$ if $s<\tau$. If $s=\tau$ we may assume without loss of generality that the controls $\Delta B_{\tau}^{+}=\Delta B_{\tau}=0$ hence $V_{\tau}=V_{\tau+}$ as we consider the supremum given in (4.25). Therefore, we have

$$
\begin{aligned}
& {\left[f\left(V_{s_{-}}+\operatorname{diag}\left(V_{s_{-}}\right) \Delta Y_{s}+\Delta B_{s}^{+}+\Delta B_{s}\right)-f\left(V_{s_{-}}+\operatorname{diag}\left(V_{s_{-}}\right) \Delta Y_{s}\right)\right] I_{s \leq \tau} } \\
\leq & -\epsilon\left(\left|\Delta B_{s}^{+}\right|+\left|\Delta B_{s}\right|\right) I_{s \leq \tau} .
\end{aligned}
$$

Therefore, by Equality (4.26), we deduce that

$$
\mathbb{E} \bar{X}_{t \wedge \tau}^{f, n} \leq f(x)-\varepsilon e^{-\beta t}(t \wedge \tau)-e^{-\beta t} \mathbb{E} Z_{t}
$$

where

$$
\begin{aligned}
Z_{t} & :=\int_{0}^{t \wedge \tau} r\left(c_{s}, f^{\prime}\left(V_{s_{-}}\right)\right) d s+\epsilon \int_{0}^{t \wedge \tau}\left|\dot{B}_{s}^{c}\right| d\left\|B^{c}\right\|_{s}+\epsilon \sum_{s \leq t \wedge \tau}\left(\left|\Delta B_{s}^{+}\right|+\left|\Delta B_{s}\right|\right), \\
r(c, p) & :=U^{*}(p)+p c-U(c) .
\end{aligned}
$$

Recall that $U^{*}(p)=\sup _{x \in C}(U(x)-p x) \geq 0$ since $U(0)=0$. Moreover, by assumption,

$$
\inf _{p \in f^{\prime}\left(\overline{\mathcal{O}_{r}}(x)\right), c \in \mathcal{C},|c|=1} p c \geq \varepsilon
$$

Since $U(c) /|c| \rightarrow 0$ as $|c| \rightarrow \infty$, we finally deduce that there exists a constant $\kappa>1$ such that

$$
\left.\inf _{p \in f^{\prime}\left(\overline{\mathcal{O}_{r}}(x)\right)} r(c, p)\right) \geq \kappa^{-1}|c|, \quad \forall c \in \mathcal{C}, \quad|c| \geq \kappa
$$

Therefore,

$$
\int_{0}^{t \wedge \tau} r\left(c_{s}, f^{\prime}\left(V_{s_{-}}\right)\right) d s \geq \kappa^{-1} \int_{0}^{t \wedge \tau} I_{\left|c_{s}\right| \geq \kappa}\left|c_{s}\right| d s .
$$

Moreover, the second integral defining $Z$ dominates $\kappa_{1}\left\|B^{c}\right\|_{t \wedge \tau}$ for some $\kappa_{1}>0$. Indeed, recall that all norms in $\mathbf{R}^{d}$ are equivalent, in particular $c^{-1}|\cdot| \leq|\cdot| 1 \leq c|$.$| for$ some $c>0$ where $|x|_{1}:=\sum_{i=1}^{d}\left|x_{i}\right|$ and $|$.$| is the Euclidean norm. It follows that$

$$
c^{-1}\left\|B^{c}\right\| \leq \operatorname{Var} B^{c} \leq c\left\|B^{c}\right\|,
$$

where $\operatorname{Var} B^{c}$ is the total variation of $B^{c}$ with respect to $|\cdot|_{1}$. At last, we have:

$$
\left|\dot{B}^{c}\right|_{1}=\sum_{i=1}^{d}\left|\dot{B}^{c i}\right|=\sum_{i=1}^{d}\left|\frac{d B^{c i}}{d\left\|B^{c}\right\|}\right|=\sum_{i=1}^{d}\left|\frac{d B^{c i}}{d \operatorname{Var} B^{c i}}\right| \frac{d \operatorname{Var} B^{c i}}{d\left\|B^{c}\right\|}=\frac{d \operatorname{Var} B^{c}}{d\left\|B^{c}\right\|} .
$$


The claimed property follows. We deduce some constant $\gamma>0$ such that

$$
\mathbb{E} X_{t \wedge \tau}^{f} \leq f(x)-e^{-\beta t} \gamma^{-1} \mathbb{E} \widetilde{Z}_{t},
$$

where

$$
\widetilde{Z}_{t}:=t \wedge \tau+\int_{0}^{t \wedge \tau} I_{\left|c_{s}\right| \geq \kappa}\left|c_{s}\right| d s+\|B\|_{t \wedge \tau+}
$$

Observe that

$$
\widetilde{Z}_{t} \geq t \wedge \tau+\|B\|_{t \wedge \tau+}-\int_{0}^{t \wedge \tau} I_{\left|c_{s}\right| \leq \kappa}\left|c_{s}\right| d s \geq(1-\kappa) t \wedge \tau+\|B\|_{t \wedge \tau+} .
$$

Using the stochastic formula of $V$, we immediately get the existence of a number $t_{0}>0$ and a measurable set $\Gamma$ with $\mathbb{P}(\Gamma)>0$ on which

$$
\left|V_{t}^{\pi, x}-x\right| \leq r / 2+\delta\|B\|_{t+}, \quad t \in\left[0, t_{0}\right],
$$

whatever the control $\pi=(B, C)$. Diminishing $t_{0}$, we may assume without loss of generality that $\kappa t_{0} \leq r /(4 \delta)$. For any $t \leq t_{0}$, the inequality $\|B\|_{\tau+} \geq r /(2 \delta)$ holds on the set $\Gamma \cap\{\tau \leq t\}$. Therefore, if $t \leq t_{0}$,

$$
\widetilde{Z}_{t} \geq(1-\kappa) \tau+2 \kappa t_{0} \geq \kappa t_{0} \geq t .
$$

On the set $\Gamma \cap\{\tau>t\}$, the inequality $\widetilde{Z}_{t} \geq t$ obviously holds. Thus, $\mathbb{E} \widetilde{Z}_{t} \geq t \mathbb{P}(\Gamma)$ if $t \in\left[0, t_{0}\right]$ and the result is proven.

Observe that, if $n$ is large enough, $\tau^{\pi} \leq \theta^{n}$ hence $X_{t \wedge \tau^{\pi}}^{f, n}$ does not depend on $n$.

Lemma 4.11 Suppose that $W$ is continuous on int $K$. Then, for any stopping time $\tau \in \mathcal{T}_{f}$, we have:

$$
W(x) \geq \sup _{\pi \in \mathcal{A}_{x}} E\left(J_{\tau}^{\pi}(x)+e^{-\beta \tau} W\left(V_{\tau+}^{x, \pi}\right) I_{\tau<\theta}\right) .
$$

Proof. The proof is an adaptation of the proof of [4, Lemma 9.2]. In the latter, it suffices to replace $\rho$ by $\rho:=\inf \left\{j \geq 1: V_{\tau+}^{x, \pi} \in \mathcal{O}_{j}\right\}$.

Lemma 4.12 Let $\mathcal{T}_{f}$ be the set of finite stopping times. Then,

$$
W(x) \leq \sup _{\pi \in \mathcal{A}_{x}} \inf _{\tau \in \mathcal{T}_{f}} E\left(J_{\tau}^{\pi}+e^{-\beta \tau} W\left(V_{\tau}^{x, \pi}\right) I_{\tau<\theta}\right) .
$$

Proof. This lemma is proved in [4] since it corresponds to [4, Lemma 9.1] which does not depend on the structure imposed on the controls $B$.

\section{Proof of Theorem 4.6.}

i) We adapt the proof of [4, Lemma 10.2] since the arguments of the proof are based on a strategy $\pi=(B, C)$ such that $B=0$ and, in our case, we need Lemma 4.11 to replace [4, Lemma 9.2]). In that case, $V_{t+}=V_{t}$ for all $t \geq 0$ and the Ito formula is valid as observed in Remark 4.9 but also in [4].

ii) Let $x \in \operatorname{int} K$ and $\phi \in C_{1}(K) \cap C^{2}(K)$ be a function with $\phi(x)=W(x)$ and $W \leq \phi$ on $K$. Suppose that $\phi$ is not a subsolution, i.e. there exists $x \in \operatorname{int} K$ such that 
the required inequality fails. Precisely, by continuity, suppose that $\mathcal{L} \phi \leq-\varepsilon, \varepsilon>0$, on a neighborhood $\overline{\mathcal{O}_{r^{\prime}}(x)} \subseteq$ int $\mathrm{K}$ of $x$. By virtue of Lemma 4.10, there exists a constant $\eta:=\eta(\varepsilon)$ and an interval $\left(0, t_{0}\right]$ such that

$$
\sup _{\pi \in \mathcal{A}_{x}} \mathbb{E}\left(J_{t \wedge \tau_{r}^{\pi}}^{x, \pi}+e^{-\beta\left(t \wedge \tau_{r}^{\pi}\right)} \phi\left(V_{t \wedge \tau_{r}^{\pi}+}^{x, \pi}\right) I\left(V_{t \wedge \tau_{r}^{\pi}-}^{x, \pi}, \Delta Y_{t \wedge \tau_{r}^{\pi}}\right)\right) \leq \phi(x)-\eta t, \quad t \in\left(0, t_{0}\right],
$$

where $\tau^{\pi}:=\tau_{r}^{\pi}$ is given by (4.24). We may assume w.l.o.g. that $r=r^{\prime}$. Fix an arbitrary $t \in\left(0, t_{0}\right]$. Applying Lemma (4.12) to $V_{+}$, we deduce that there exists $\pi \in \mathcal{A}_{x}$ such that

$$
W(x) \leq \inf _{\tau \in \mathcal{T}_{f}} \mathbb{E}\left(J_{t \wedge \tau}^{x, \pi}+e^{-\beta(t \wedge \tau)} W\left(V_{t \wedge \tau+}^{x, \pi}\right) I_{t \wedge \tau<\theta}\right)+\frac{1}{2} \eta t
$$

As $W \leq \phi$ and since $I\left(V_{t \wedge \tau_{r}^{\pi}-}^{x, \pi}, \Delta Y_{t \wedge \tau_{r}^{\pi}}\right)=0$ implies that $I_{t \wedge \tau_{r}^{\pi}<\theta}=0$, we obtain from above that $W(x) \leq \phi(x)-\frac{1}{2} \eta t$, in contradiction since $W(x)=\phi(x)$.

\section{Uniqueness theorem}

Definition 5.1 We say that a positive function $\ell \in C_{1}(K) \cap C^{2}(\operatorname{int} K)$ is a Lyapunov function if the following properties are satisfied:

1) $\ell^{\prime}(x) \in \operatorname{int} K^{*}$ and $\mathcal{L}_{0} \ell(x) \leq 0$ for all $x \in \operatorname{int} K$,

2) $\ell(x) \rightarrow \infty$ as $|x| \rightarrow \infty$.

In other words, $\ell$ is a classical supersolution of the truncated equation (excluding the term $U^{*}$ ), continuous up to the boundary, and increasing to infinity at infinity. Let us introduce the following condition on $\Pi$ which guaranties the uniqueness of the HJB equation we consider, under the condition that there exists a Lyapunov function as stated in the next theorem.

Condition $\Pi^{0}: \forall x \in \operatorname{int} K, \Pi(z: x+\operatorname{diag} x z \in \partial K)=0$.

Remark 5.2 This condition holds in the two dimensional case if the first component of the underlying asset is a bond $B=1$ so that $\Pi=\delta_{0} \otimes \pi$ where we suppose that $\pi$ does not charge the singletons.

Theorem 5.3 Suppose that there exists a Lyapunov function $\ell$ and the Lévy measure $\Pi$ is such that

$$
\left.\Pi\left(z: \hat{x}+D_{\hat{x}} z \in \partial K\right\}\right)=0 \quad \forall \hat{x} \in \operatorname{int} K .
$$

Then the Dirichlet problem (4.12) has at most one viscosity solution in the class of continuous functions satisfying the growth condition

$$
W(x) / \ell(x) \rightarrow 0, \quad|x| \rightarrow \infty
$$

Moreover, $W$ is concave. 


\subsection{Proof of Theorem 5.3}

Uniqueness is an immediate consequence of Theorem 5.4 below. Moreover, Theorem 5.5 ensures that the unique solution $W$ is concave. Notice that Proposition 8.34 ensures the existence of a Lyapunov function under mild assumptions.

Theorem 5.4 (Maximum principle) Suppose that there exists a Lyapunov function $\ell$ and $\Pi$ satisfies Condition $\Pi^{0}$. Let $\hat{K}$ be a nonempty subset of $K$ with nonempty interior. Let $W$ be a continuous viscosity subsolution of the Dirichlet problem (4.12) on $\hat{K}$ satisfying the growth condition

$$
|W(x)| / \ell(x) \rightarrow 0, \quad|x| \rightarrow \infty .
$$

Let $\tilde{W}$ be a continuous viscosity supersolution of the Dirichlet problem (4.12) on $\tilde{K}$ such that $\tilde{W} \geq W$ on $\partial \hat{K}$. Suppose that either $\tilde{W} \geq 0$ or $\tilde{W}$ satisfies (5.31). Then, $\tilde{W} \geq W$ on $\hat{K}$.

Proof. The proof is an easy adaptation of the proof of [4, Theorem 11.2 ]. Indeed, we follow the same reasoning but we consider the supremum of $\Delta_{n}$ on the set $\hat{K}$.

Theorem 5.5 If the HJB equation (4.12) admits a unique global viscosity solution in $C_{0}(K)$, then the Bellman function is concave.

Proof. Consider the (modified) Bellman function $\widehat{W}$ only defined by the (non empty) class of admissible strategies generating the portfolio processes evolving in (int $K) \cup\{0\}$ on $\mathbf{R}_{+}$, i.e

$$
\widehat{\mathcal{A}}_{x}:=\left\{\pi \in \mathcal{A}: V_{t}^{\pi} \in(\operatorname{int} K) \cup\{0\}, \forall t \geq 0\right\} \neq \emptyset,^{1}
$$

and

$$
\widehat{W}(x):=\sup _{\pi \in \widehat{\mathcal{A}}_{x}} E J_{\infty}^{\pi}(x), \quad x \in \operatorname{int} K,
$$

We also consider the corresponding stopping times

$$
\theta=\theta^{x, \pi}:=\inf \left\{t: V_{t}^{x, \pi} \notin \operatorname{int} K\right\}
$$

for $\pi \in \widehat{\mathcal{A}}_{x}$ and we have $V_{t}^{\pi}=0$ for $t \geq \theta$. Therefore, the consumption strategy $c$ is zero after $\theta$. We deduce that the Bellman function $\widehat{W}$ is a global viscosity solution to the same HJB equation as $W$. Indeed, we use Lemma 4.12 or equivalently [4, Lemma 9.1] and we adapt [4, Lemma 9.2], where we replace the random variable $\rho$ in the proof of [4, Lemma 9.2] by $\rho:=\inf \left\{j \geq 1: V_{\tau+}^{x, \pi} \in \mathcal{O}_{j}\right\}$ and the strategy $\tilde{\pi}$ is replaced by

$$
\tilde{\pi}:=\pi I_{\left[0, \tau_{k}\right)}+\sum_{n=1}^{\infty}\left[\left(y_{n}-V_{\tau_{k}-}^{x, \pi}, 0\right)+\bar{\pi}^{n, k}\right] I_{\left[\tau_{k}, \infty[\right.} I_{\{\rho=n\}} I_{\left\{V_{\tau_{k}-}^{x, \pi}-y_{n} \in K\right\}} I_{\left\{\tau_{k}<\theta\right\}}
$$

For $t<\tau_{k}$, it is clear that $V_{t}^{\tilde{\pi}} \in($ int $K) \cup\{0\}$ as $\pi \in \widehat{\mathcal{A}}_{x}$. Moreover, on $\{\rho=n\}, V_{\tau_{k}}^{\tilde{\pi}}=$ $y_{n} \in \operatorname{int} K$ provided that $V_{\tau_{k}-}^{x, \pi}-y_{n} \in K$ and $\tau_{k}<\theta$. Otherwise, $V_{\tau_{k}}^{x, \tilde{\pi}}=V_{\tau_{k}-}^{x, \pi}=0$ by Lemma 4.7. It follows that $\tilde{\pi} \in \widehat{\mathcal{A}}_{x}$ and we may conclude as in [4, Lemma 9.2] since $u \geq 0$.

By assumption, the global viscosity solution of this HJB equation is unique hence $\widehat{W}=W$. It is well known that the function $\widehat{W}$ is concave (the proof of it is given in Framstad et al. [6]). Therefore, $W$ is also concave.

1 Observe that $\widehat{\mathcal{A}}_{x} \neq \emptyset$. Indeed, rebalance the portfolio starting from $x \in \operatorname{int} K$ so that $V_{+}=0$. 


\section{Finiteness of the value function}

We denote by $\Phi$ the set of all continuous functions $f: K \mapsto \mathbf{R}_{+}$increasing with respect to the partial ordering $\succeq_{K}$ and such that for every $x \in \operatorname{int} K$ and $\pi=(B, C)$ the positive process $X_{t}^{f}=e^{-\beta t \wedge \theta} f\left(V_{t+}^{\theta}\right)+J_{t}^{\pi}, t \geq 0$, is a supermartingale. The following lemma (see also [4]) is a consequence of Lemma 4.8:

Lemma 6.1 Let $f \in C^{2}\left(\mathbf{R}^{d}\right)$ be a non negative classical supersolution of (4.12) which vanishes outside int $K$, then $f \in \Phi$.

Proof. First, we claim that a classical supersolution is increasing with respect to the partial ordering on $K$. Indeed, by the finite increments formula, for any $x, h \in \operatorname{int} K$,

$$
f(x+h)-f(x)=f^{\prime}(x+\nu h) h
$$

for some $\nu \in[0,1]$. The right-hand side is non negative because for the supersolution $f$ we have the inequality $\Sigma_{G}\left(f^{\prime}(y)\right) \leq 0$ whatever is $y \in \operatorname{int} K$, or, equivalently, $f^{\prime}(y) h \geq$ 0 for all $h \in K$. As a classical supersolution of (4.12), $f$ satisfies the inequality

$$
\mathcal{L}_{0} f\left(V_{u_{-}}\right)-f^{\prime}\left(V_{u_{-}}\right) c_{u}+U\left(c_{u}\right) \leq 0, \quad \forall u<\theta .
$$

By Remark 4.9, as $\left(N_{t}^{\theta^{n}}\right)_{t \geq 0}$ is a local martingale bounded from below by $-f(x)$, the local martingale $N_{t}:=\lim _{n \rightarrow \infty} N_{t}^{\theta^{n}}$ is also bounded from below. Therefore, it is a supermartingale. In particular, $N_{t}$ is integrable and so is the non positive process

$$
R+\int_{0}^{. \wedge \theta} e^{-\beta u}\left[\mathcal{L}_{0} f\left(\widetilde{V}_{u_{-}}\right)-f^{\prime}\left(\widetilde{V}_{u_{-}}\right) c_{u}+U\left(c_{u}\right)\right] d u
$$

We then conclude that $\left(X_{t}^{f}\right)$ is a supermartingale.

The following proposition formulates finiteness and continuity up to boundary $\partial K$ of the Bellman function in term of $\Phi$. This corresponds to [4, Lemma 8.1].

\section{Proposition 6.2}

a) If $f \in \Phi$, then $W \leq f$ on $K$. Hence, if $\Phi \neq \emptyset$, then $W$ is finite.

b) If $x_{0} \in \partial K$ is such that, for every $\epsilon>0$, there exists $f_{\epsilon} \in \Phi$ with $f_{\epsilon}\left(x_{0}\right) \leq \epsilon$, then $W$ is continuous at $x_{0}$ and $W\left(x_{0}\right):=0$.

Corollary 6.3 Let $f \in C^{2}\left(\mathbf{R}^{d}\right)$ be a non negative classical supersolution of (4.12) which vanishes outside int $K$, then $W$ is finite.

Proof. By Lemma 6.1, $\Phi \neq \emptyset$. We conclude by Proposition 6.2 .

\section{Continuity of the value function}

Lemma 7.1 Let us consider $x_{0} \in \operatorname{int} \mathrm{K}$. Then, $\limsup _{\lambda \rightarrow 1} W\left(\lambda x_{0}\right) \leq W\left(x_{0}\right)$. 
Proof. We may find $\tilde{\pi}^{n}=\left(\tilde{B}^{n}, \tilde{C}^{n}\right) \in \mathcal{A}_{\lambda x_{0}}$ depending on $\lambda$ such that

$$
W\left(\lambda x_{0}\right) \leq E \int_{0}^{\theta^{\pi^{n}}} e^{-\beta s} U\left(\tilde{c}_{s}^{n}\right) d s+\frac{1}{n} .
$$

Observe that, if $\tilde{\pi}^{n}=\left(\tilde{B}^{n}, \tilde{C}^{n}\right) \in \mathcal{A}_{\lambda x_{0}}$, then $\tilde{\pi}^{n} / \lambda:=\left(\tilde{B}^{n} / \lambda, \tilde{C}^{n} / \lambda\right) \in \mathcal{A}_{x_{0}}$ and $\theta^{\tilde{\pi}^{n} / \lambda}=\theta^{\tilde{\pi}^{n}}$. We rewrite $\tilde{\pi}^{n}$ as $\tilde{\pi}^{n}=\lambda \pi^{n}$ where $\pi^{n}:=\left(B^{n}, C^{n}\right) \in \mathcal{A}_{x_{0}}$. By (7.32), we deduce that

$$
W\left(\lambda x_{0}\right) \leq E \int_{0}^{\theta^{\pi^{n}}} e^{-\beta s} U\left(\lambda c_{s}^{n}\right) d s+\frac{1}{n} .
$$

As $\left.\lambda^{-1} \in\right] 0,1[$ and $x \mapsto U(x)$ is concave with $U(0)=0$, we get that

$$
U\left(c_{s}^{n}\right)=U\left(\lambda^{-1}\left(\lambda c_{s}^{n}\right)+\left(1-\lambda^{-1}\right) \times 0\right) \geq \lambda^{-1} U\left(\lambda c_{s}^{n}\right) .
$$

We then get $W\left(\lambda x_{0}\right) \leq \lambda W\left(x_{0}\right)+n^{-1}$ and the conclusion follows as $n \rightarrow \infty$. $\square$

Lemma 7.2 We have $V_{t_{-}} \in \operatorname{int} K$ if $t \in[0, \theta[$.

Proof. Suppose that $V_{t-} \in \partial K$ for some $t<\theta$. Then, by Lemma 4.7 and Assumption $5)$ of the model, we have $\Delta Y_{t}=\Delta B_{t}=\Delta C_{t}=0$. Therefore, $V_{t}=V_{t_{-}} \in \partial K$ hence a contradiction.

Note that we also deduce from Lemma 4.7 that the portfolio process exits $K$ either in a continuous manner (in the case $V_{\theta_{-}} \in \partial K$ ) or due to a jump (in the case $V_{\theta_{-}} \in$ int $K)$.

Lemma 7.3 Let us consider a sequence $x_{n} \rightarrow x_{0} \in \operatorname{int} K$ and $T \in(0, \infty)$. Then, for any $\pi \in \mathcal{A}_{x_{0}}$, the sequence of portfolios $V^{(n)}=V^{\pi, x_{n}}$ with initial values $V_{0_{-}}^{(n)}=x_{n}$ is such that

$$
T \wedge \theta \leq \liminf _{n} \theta_{n} \wedge T
$$

where $\theta, \theta_{n}$ are the stopping times defined by $V:=V^{\pi, x}, V^{(n)}$ respectively in (2.4).

Proof. Note that $V^{(n)}$ and $V$ are uniquely defined by (2.3). Moreover, $\widetilde{V}^{n}:=V^{(n)}-V$ satisfies the dynamics

$$
d \widetilde{V}_{t}^{n i}=\widetilde{V}_{t_{-}}^{n i} d Y_{t}^{i}, \quad \widetilde{V}_{0-}^{n}=x_{n}-x_{0} .
$$

Hence $\widetilde{V}_{t}^{n i}=\left(x_{n}^{i}-x_{0}^{i}\right) S_{t}^{i}$ or equivalently $\widetilde{V}_{t}^{n}=\operatorname{diag}\left(\left(x_{n}-x_{0}\right) / S_{0}\right) S_{t}$. We deduce that

$$
\sup _{t \leq T}\left|V_{t}^{(n)}-V_{t}\right| \leq \frac{\left|x_{n}-x_{0}\right|}{\left|S_{0}\right|} S_{T}^{*}
$$

where $S_{T}^{*}:=\sup _{t \leq T}\left|S_{t}\right|$. On the other hand, if $\delta>0$ is small enough, we may write

$$
\inf _{s \leq T \wedge \theta-\delta} d\left(V_{s} ; \partial K\right)=\lim _{n} d\left(V_{s_{n}} ; \partial K\right),
$$

where $s_{n} \in[0, T \wedge \theta-\delta]$ converges to $s_{0} \in[0, T \wedge \theta-\delta]$ by compactness argument. First assume that $s_{n} \uparrow s_{0}$. Then,

$$
\inf _{s \leq T \wedge \theta-\delta} d\left(V_{s} ; \partial K\right)=d\left(V_{s_{0_{-}}} ; \partial K\right)>0,
$$


by virtue of Lemma 7.2. Otherwise, we get that

$$
\inf _{s \leq T \wedge \theta-\delta} d\left(V_{s} ; \partial K\right)=d\left(V_{s_{0+}} ; \partial K\right)>0 .
$$

Therefore, there exists $\epsilon>0$ such that

$$
d\left(V_{s} ; \partial K\right)>\epsilon, \quad \forall s \in[0, T \wedge \theta-\delta] .
$$

Applying the triangular inequality $d\left(V_{s} ; \partial K\right) \leq d\left(V_{s} ; V_{s}^{n}\right)+d\left(V_{s}^{n} ; \partial K\right)$ we then deduce that

$$
d\left(V_{s}^{n} ; \partial K\right)>\epsilon-d\left(V_{s} ; V_{s}^{n}\right) \geq \epsilon-\frac{\left|x_{n}-x_{0}\right|}{\left|S_{0}\right|} S_{T}^{*}, \quad s \in[0, T \wedge \theta-\delta] .
$$

It follows that for $n$ large enough

$$
d\left(V_{s}^{n} ; \partial K\right)>0, \quad s \in[0, T \wedge \theta-\delta] .
$$

We deduce that $\theta^{n} \geq T \wedge \theta-\delta$ if $n$ is large enough. We have shown that whatever $\delta>0$ is small enough, there exists a.s. $n_{0}$ such that, for every $n \geq n_{0}, T \wedge \theta-\delta \leq \theta^{n}$. Therefore, $T \wedge \theta-\delta \leq \inf _{n \geq n_{0}} \theta^{n}$ and finally $T \wedge \theta \leq \liminf _{n} \theta_{n} \wedge T$.

Theorem 7.4 Assume that $W\left(x_{0}\right)<\infty$ where $x_{0} \in \operatorname{int} K$. Then, $W$ is continuous at $x_{0}$.

Proof. It suffices to show that $W$ is both upper semicontinuous and lower semicontinuous.

- Let us first show that $\lim \sup _{x \rightarrow x_{0}} W(x) \leq W\left(x_{0}\right)$. In the contrary case, we have $\limsup _{x \rightarrow x_{0}} W(x)>W\left(x_{0}\right)$. Note that $\limsup _{x \rightarrow x_{0}} W(x)=\lim _{k} W\left(x_{k}\right)$ where $x_{k}$ is a subsequence converging to $x_{0}$. As $x_{0} \in \operatorname{int} K$, we may assume that $x_{k} \in \operatorname{int} K$. We define $\widetilde{x}_{k}=\left(1+k^{-1}\right) x_{0} \in \operatorname{int} K$ such that $\widetilde{x}_{k} \in x_{0}+\operatorname{int} K$ if $k$ is large enough. As $x_{k} \rightarrow x_{0}$ and $\widetilde{x}_{k}-$ int $K$ is an open set containing $x_{0}$, there exists a subsequence $x_{n_{k}}$ such that $x_{n_{k}} \in \widetilde{x}_{k}-\operatorname{int} K$ hence $x_{n_{k}} \preceq \widetilde{x}_{k}$. Since $W$ is increasing with respect to $\succeq$, we obtain that $\lim \sup _{k} W\left(\widetilde{x}_{k}\right) \geq \lim _{k} W\left(x_{n_{k}}\right)>W\left(x_{0}\right)$. On the other hand, by virtue of Lemma $7.1, W\left(\widetilde{x}_{k}\right) \leq W\left(x_{0}\right)$ hence a contradiction.

- Let us show that $\liminf x_{x_{n} \rightarrow x_{0}} W\left(x_{n}\right) \geq W\left(x_{0}\right)$. For an arbitrary $\epsilon>0$, there exists $\pi \in \mathcal{A}_{x_{0}}$ such that

$$
W\left(x_{0}\right) \leq \epsilon+\mathbb{E} \int_{0}^{\theta^{\pi}} e^{-\beta s} U\left(c_{s}\right) d s .
$$

We then deduce $T \in(0, \infty)$ such that

$$
W\left(x_{0}\right) \leq 2 \epsilon+\mathbb{E} \int_{0}^{\theta^{\pi} \wedge T} e^{-\beta s} U\left(c_{s}\right) d s .
$$

We introduce the stopping times $\theta^{n}$ associated to the portfolios defined by the strategy $\pi$ and the initial values $x_{n}$. Observe the inequality

$$
I_{s<\lim \inf _{n} \theta^{n} \wedge T} \leq \liminf _{n} I_{s \leq \theta^{n} \wedge T} .
$$


So, by virtue of Lemma 7.3 and Fatou's lemma, we then deduce that

$$
\begin{aligned}
& W\left(x_{0}\right) \leq 2 \epsilon+\liminf _{n} \mathbb{E} \int_{0}^{\theta^{n} \wedge T} e^{-\beta s} U\left(c_{s}\right) d s, \\
& W\left(x_{0}\right) \leq 2 \epsilon+\liminf _{n} W\left(x_{n}\right) .
\end{aligned}
$$

Since $\epsilon>0$ is arbitrarily chosen, we deduce that $W\left(x_{0}\right) \leq \liminf _{n} W\left(x_{n}\right)$.

\section{Existence of Lyapunov functions}

In this subsection, we study the existence of Lyapunov functions. We only focus on the case where the matrix $A=\left(a_{i j}\right)$ is diagonal with $a_{i i}=\sigma^{i}$, such that $\sigma^{0}=0, \mu^{0}=0$ and $\sigma^{i} \neq 0, i=1 \ldots d$, i.e. the first asset is a numéraire and the others are risky assets. We also suppose that the utility function is $U(x)=u_{\gamma}\left(x e_{0}\right)$, where $u_{\gamma}(t)=t^{\gamma} / \gamma, \gamma \in$ $(0,1)$, and $\mathcal{C}=\mathbf{R}_{+} e_{0}$. In this subsection we work under the following condition

$$
\int_{\mathbf{R}^{d}}|z| \Pi(d z)<\infty .
$$

For $p \in \operatorname{int} K^{*}$, we construct a Lyapunov function of the form $v(x):=u_{\eta}(p x)$, where $u_{\eta}(x)=\frac{x^{\eta}}{\eta}$ and $\gamma<\eta<1$. Note that the Bellman function is homogeneous of degree $\gamma$. Therefore, we choose $\eta \geq \gamma$ so that the Lyapunov function is growing faster than the Bellman function. If such a Lyapunov exists, the HJB equation admits a unique concave solution in $C^{1}$ by Theorem 5.3.

We have $u_{\eta}^{\prime}(x)=(p x)^{\eta-1} p \in \operatorname{int} K^{*}$ as required for $v$ to be a Lyapunov function. Moreover,

$$
\begin{aligned}
\mathcal{L}_{0} v(x) & =\frac{1}{2}\langle A(x) p, p\rangle u_{\eta}^{\prime \prime}(p x)+\langle\mu(x), p\rangle u_{\eta}^{\prime}(p x)-\beta u_{\eta}(p x) \\
& +\int_{\mathbf{R}^{d}}\left[u_{\eta}\left(p x+{ }^{t} p \operatorname{diag}(x) z\right) I(x, z)-u_{\eta}(p x)-u_{\eta}^{\prime}(p x)^{t} p \operatorname{diag}(x) z\right] \Pi(d z) .
\end{aligned}
$$

Let us denote the integral expression above by $H_{\eta}(x)$. Our goal is to choose $u$ such that $\mathcal{L}_{0} v(x) \leq 0$ on $\operatorname{int} K$, or equivalently

$$
\beta \geq \sup _{x \in K}\left[p \operatorname{diag}(x) \mu \frac{u_{\eta}^{\prime}(p x)}{u_{\eta}(p x)}+\frac{1}{2}\langle A(x) p, p\rangle \frac{u_{\eta}^{\prime \prime}(p x)}{u_{\eta}(p x)}+\frac{H_{\eta}(x)}{u_{\eta}(p x)}\right] .
$$

Let us introduce

$$
L_{\eta}(p, x)=p \operatorname{diag}(x) \mu \frac{u_{\eta}^{\prime}(p x)}{u_{\eta}(p x)}+\frac{1}{2}\langle A(x) p, p\rangle \frac{u_{\eta}^{\prime \prime}(p x)}{u_{\eta}(p x)} .
$$

We have

$$
\begin{aligned}
L_{\eta}(p, x) & =\frac{1}{2} \frac{\eta}{1-\eta} \sum_{i=1}^{d} \frac{\mu_{i}^{2}}{\sigma_{i}^{2}}-\frac{1}{2} \eta(1-\eta) \sum_{i=1}^{d}\left(\frac{\sigma_{i} p_{i} x_{i}}{p x}-\frac{\mu_{i}}{\sigma_{i}(1-\eta)}\right)^{2}, \\
& \leq \frac{1}{2} \frac{\eta}{1-\eta} \sum_{i=1}^{d} \frac{\mu_{i}^{2}}{\sigma_{i}^{2}} .
\end{aligned}
$$


We then choose $\beta$ such that

$$
\beta \geq \frac{1}{2} \frac{\eta}{1-\eta} \sum_{i=1}^{d} \frac{\mu_{i}^{2}}{\sigma_{i}^{2}}+\sup _{x \in K} \frac{H_{\eta}(x)}{u_{\eta}(p x)} .
$$

Put $k(p, x)=\frac{1}{p x} p \operatorname{diag}(x)$. We have

$$
\frac{H_{\eta}(x)}{u_{\eta}(p x)} \leq \int_{\mathbf{R}^{d}}\left[(1+k(p, x) z)^{\eta} 1_{\{1+k(p, x) z>0\}}-1-\eta k(p, x) z\right] \Pi(d z) .
$$

Note that $k(p, t x)=k(p, x)$ for $t>0$. Therefore, instead of considering the r.h.s of the equality above on $K$, we may simply consider it on $\mathcal{B}_{1}:=K \cap \mathcal{O}_{1}$, where $\mathcal{O}_{1}$ is the unit ball $\{x:|x|=1\}$. It is easy to prove that this expression is bounded on $\mathcal{B}_{1}$. We then define

$$
h(\eta):=\inf _{p \in \operatorname{int} K^{*}} \sup _{x \in \mathcal{B}_{1}} \int_{\mathbf{R}^{d}}\left[(1+k(p, x) z)^{\eta} 1_{\{1+k(p, x) z>0\}}-1-\eta k(p, x) z\right] \Pi(d z),
$$

and we chose $\beta$ such that

$$
\beta>\frac{1}{2} \frac{\eta}{1-\eta} \sum_{i=1}^{d} \frac{\mu_{i}^{2}}{\sigma_{i}^{2}}+h(\eta) .
$$

We may deduce the following:

\section{Proposition 8.1}

i) If the condition (8.33) holds, then $v(x)=\frac{(p x)^{\eta}}{\eta}, 1>\eta>\gamma$ is a Lyapunov function with respect to $\mathcal{L}_{0}$ for some $p \in K^{*}$ with $p^{1}=1$.

ii) Set $\bar{h}(\gamma):=\lim \inf h(\eta)$ and suppose that

$$
\beta>\frac{1}{2} \frac{\gamma}{1-\gamma} \sum_{i=1}^{d} \frac{\mu_{i}^{2}}{\sigma_{i}^{2}}+\bar{h}(\gamma) .
$$

Then, the HJB equation admits a unique solution under the conditions of Theorem 5.3.

\section{Classical supersolution}

The hypothesis of this section are those of Section 8 and the notations are the same. By Lemma 6.1, if we construct a non negative classical supersolution $f \in C^{2}\left(\mathbf{R}^{d}\right)$ of (4.12) which vanishes outside int $K$, then $f \in \Phi$. Therefore, by Proposition $6.2, W$ is finite.

Let us define $v(x):=k u_{\gamma}(p x)$. By definition,

$$
\begin{aligned}
U^{*}\left(v^{\prime}(x)\right) & =\sup _{y \in \mathcal{C}}\left(U(y)-v^{\prime}(x) y\right)=\sup _{y \in \mathcal{C}}\left(U(y)-k u_{\gamma}^{\prime}(p x) p y\right), \\
& =\sup _{y_{1} \geq 0}\left(\frac{\left(y_{1}\right)^{\gamma}}{\gamma}-k(p x)^{\gamma-1} p_{1} y_{1}\right)
\end{aligned}
$$


Then, with $p_{1}=1, U^{*}\left(v^{\prime}(x)\right)=k^{\frac{\gamma}{\gamma-1}}(p x)^{\gamma}(1 / \gamma-1)$. Since $u^{\prime} \geq 0, G \subseteq-K$, and $p \in K^{*} \backslash\{0\}$, we have

$$
\Sigma_{G}\left(v^{\prime}(x)\right)=\sup _{x \in G} u_{\gamma}^{\prime}(p . x) p x \leq 0 .
$$

Our goal is to choose $p, k$ so that, on int $K$, we have

$$
\begin{aligned}
& k^{\frac{\gamma}{\gamma-1}}(p x)^{\gamma}(1 / \gamma-1)+\frac{k}{2}\langle A(x) p, p\rangle u_{\gamma}^{\prime \prime}(p x)+k\langle\mu(x), p\rangle u_{\gamma}^{\prime}(p x)-k \beta u_{\gamma}(p x) \\
& +\int_{\mathbf{R}^{d}}\left[u_{\gamma}\left(p x+{ }^{t} p \operatorname{diag}(x) z\right) I(x, z)-u_{\gamma}(p x)-u_{\gamma}^{\prime}(p x)^{t} p \operatorname{diag}(x) z\right] \Pi(d z) \leq 0 .
\end{aligned}
$$

Adapting the reasoning of the previous subsection, we choose $\beta$ such that

$$
\beta>\frac{1}{2} \frac{\gamma}{1-\gamma} \sum_{i=1}^{d} \frac{\mu_{i}^{2}}{\sigma_{i}^{2}}+h(\gamma)+k^{\frac{1}{\gamma-1}}(1-\gamma)
$$

\section{Proposition 9.1}

i) Suppose that Condition (9.35) holds. Then, the function

$$
v(x)=\frac{k}{\gamma}(p x)^{\gamma},
$$

is a classical supersolution to the HJB equation for some $p \in K^{*}$ with $p^{1}=1$.

ii) In the two-dimensional model with the power utility function, assume that the Merton parameter satisfies

$$
\kappa_{M}:=\frac{1}{1-\gamma}\left(\beta-\frac{\gamma \mu^{2}}{2 \sigma^{2}(1-\gamma)}-h(\gamma)\right)>0 .
$$

Then, there exists $p \in K^{*}$ with $p^{1}=1$ such that the function $f(x)=m(p x)^{\gamma}$ is a classical supersolution of the HJB equation and $m>(1 / \gamma) \kappa_{M}^{\gamma-1}$.

Combining Propositions 8.1 and 9.1, we obtain the following result

Corollary 9.2 Let $h^{*}$ be defined as $h^{*}(\gamma):=\max (h(\gamma), \bar{h}(\gamma))$. If

$$
\beta>\frac{1}{2} \frac{\gamma}{1-\gamma} \sum_{i=1}^{d} \frac{\mu_{i}^{2}}{\sigma_{i}^{2}}+h^{*}(\gamma)
$$

there exists a classical supersolution to the HJB equation and a Lyapunov function with higher growing order than the Bellman function one. 


\section{Application to the two-asset model}

We investigate the properties satisfied by the Bellman function and we construct an optimal policy in the case $d=2$. We use the notation $z=(x, y)^{\prime}=\left(z_{1}, z_{2}\right)^{\prime}$ to designate a generic element $z$ of $\mathbf{R}^{2}$ where' ${ }^{\prime}$ is the transpose operator. The canonical basis of $\mathbf{R}^{2}$ is $\left(e_{1}, e_{2}\right)$ where $e_{1}=(1,0)^{\prime}$ and $e_{2}=(0,1)^{\prime}$. The risk-free asset (a Bond) is supposed to be a constant, and the risky asset follows a geometric Lévy process:

$$
\begin{aligned}
& d S_{t}^{1}=0, S_{0}^{1}=1, \\
& d S_{t}^{2}=S_{t-}^{2}\left(\mu d t+\sigma d W_{t}+\int_{\mathbf{R}} x(p(d y, d t)-q(d y, d t))\right), S_{0}^{2}=1,
\end{aligned}
$$

where $p$ is the jump measure of $S^{2}$ and $q(d y, d t)=\pi(d y) d t$ is its compensator. We suppose that $\pi(d y)$ is a positive measure concentrated in $(-1, \infty)$ which does not charge the singletons and satisfies the following condition

\section{Condition (I):}

$$
\int_{-1}^{\infty} \max (1,|t|) \pi(d t)<\infty
$$

The inequality (10.37) ensures that $\pi$ is a finite measure such that the associated Lévy process has a finite activity. This implies that

$$
Y^{1}=0, Y_{t}^{2}=\sigma W_{t}+\mu t+\sum_{i=1}^{N_{t}} \chi_{i}
$$

where $N_{t}=\sum_{n=1}^{\infty} 1_{T_{n} \leq t}$ is a Poisson process of intensity $\lambda>0$ and $\left(\chi_{i}\right)_{i \geq 1}$ is a sequence of i.i.d. $\pi$-distributed random variables independent of $N$. A portfolio process satisfies by definition the following dynamics:

$$
\begin{aligned}
& d V_{t}^{1}=d L_{t}^{21}-\left(1+\lambda^{12}\right) d L_{t}^{12}-c_{t} d t \\
& d V_{t}^{2}=V_{t-}^{2}\left(\mu d t+\sigma d W_{t}+\int_{\mathbf{R}} x(p(d y, d t)-q(d y, d t))\right)+d L_{t}^{12}-\left(1+\lambda^{21}\right) d L_{t}^{21},
\end{aligned}
$$

where $L^{i j}, i, j=1,2$, are the transfer processes supposed to be làdlàg and $\left(\lambda^{i j}\right)_{i, j=1,2}$ are the transaction costs coefficients. We rewrite the dynamics of a portfolio process under the vector form:

$$
d V_{t}=\operatorname{diag} V_{t-}\left(\tilde{\mu} d t+\tilde{\sigma} d W_{t}+\int z(\tilde{p}(d z, d t)-\tilde{\Pi}(d z) d t)\right)+d B_{t}-d C_{t} .
$$

In the following, we use the notations $\tilde{\mu}=(0, \mu)^{\prime}, \tilde{\sigma}=(0, \sigma)^{\prime}$ and, with $z=(x, y)^{\prime}$,

$$
\tilde{p}(d z, d t)=\delta_{0}(d x) d t \otimes \delta_{\Delta Y_{t}}(d y) d N_{t}, \quad \tilde{\Pi}(d z)=\lambda \delta_{0}(d x) \otimes \pi(d y) .
$$

This means that $A=\operatorname{diag}\left(0, \sigma^{2}\right)$ is the diagonal matrix with diagonal elements 0 and $\sigma^{2}$. Morever, we introduce $d C_{t}=\left(c_{t} d t, 0\right)^{\prime}$ and

$$
d B_{t}=\left(d L_{t}^{21}-\left(1+\lambda^{12}\right) d L_{t}^{12}, d L_{t}^{12}-\left(1+\lambda^{21}\right) d L_{t}^{21}\right)^{\prime} .
$$


The optimization problem reads as

$$
\mathbb{E} \int_{0}^{\theta} e^{-\beta s} u\left(c_{s}\right) d s \rightarrow \max
$$

where $u: \mathbf{R}_{+} \rightarrow \mathbf{R}$ is a concave utility function. In the sequel, we consider the case of power utility functions, i.e. $u(r)=\frac{r^{\gamma}}{\gamma}, \gamma \in(0,1)$. Therefore, $W$ is homogeneous of degree $\gamma$ :

$$
W(\nu x)=\nu^{\gamma} W(x), \quad \forall x \in K, \nu \geq 0
$$

In this framework, the solvency cone $K$ is simply a sector generated by the vectors $g_{1}=\left(1+\lambda^{12}\right) e_{1}-e_{2}, g_{2}=\left(1+\lambda^{21}\right) e_{2}-e_{1}$. The dual cone of $K$ is $K^{*}=$ cone $\left\{p_{1}, p_{2}\right\}$ with $p_{1}=\left(1+\lambda^{12}\right) e_{2}+e_{1}, p_{2}=\left(1+\lambda^{21}\right) e_{1}+e_{2}$. For the sake of simplicity, we suppose that $\lambda^{12}=\lambda^{21}=\lambda$. The consumption region is $C=\mathbf{R}_{+} e_{1}$. Therefore, the HJB equation is given by:

$$
\begin{aligned}
& F\left(W^{\prime \prime}(z), W^{\prime}(z), H(W, z), W(z), z\right)=0, \\
& W(z)=0 \text { on } \partial K
\end{aligned}
$$

where

$$
\begin{aligned}
F_{0}(X, p, H, W, z) & :=\frac{1}{2} \sigma^{2} y X_{22}+\mu y p_{2}+H-\beta W \\
F(X, p, H, W, z) & :=\max \left\{F_{0}(X, p, H, W, z)+U^{*}(p), \Sigma_{G}(p)\right\} .
\end{aligned}
$$

By simple computation, we get that $U^{*}(p)=\frac{1}{\gamma}\left(p_{1}\right)^{\frac{\gamma}{\gamma-1}}$, if $p=\left(p_{1}, p_{2}\right)^{\prime}$. Moreover

$$
\Sigma_{G}(p) \leq 0 \Leftrightarrow \max \left\{-g_{1} p,-g_{2} p\right\} \leq 0,
$$

and

$$
H(u, z)=\int_{\mathbf{R}}\left[u(x, y(1+t)) I(z, t)-u(x, y)-u_{y}^{\prime}(x, y) y t\right] \pi(d t),
$$

where $I(z, t):=1_{\{(x, y+y t) \in \operatorname{intK}\}}, z=(x, y)^{\prime}$. We now formulate properties satisfied by the operator $H(u, z)$ :

\section{Lemma 10.1}

i) If $u \in C_{1}(K) \cap C^{1}\left(K \backslash \mathbf{R} e_{1}\right)$ is a non negative concave function such that $\Sigma_{G}\left(u^{\prime}\right) \leq 0$ on $K$ (i.e. $u$ is increasing with respect to the natural order on $K$ ), then the operator $H(u, z)$ is non positive.

ii) If $u \in C^{1}\left(K \backslash \mathbf{R} e_{1}\right)$ is homogeneous of degree $\gamma<1$ and $\pi(\mathbf{R})<\infty$ then $H(u,$. is continuous on $K \cap\{(x, y): y>0\}$. Moreover, if $u_{y}\left(x, 0^{+}\right)$exists and is finite, then so is $H\left(u,\left(x, 0^{+}\right)\right)$.

iii) If $u \in C^{2}\left(K \backslash \mathbf{R} e_{1}\right)$ is homogeneous of degree $\gamma<1$ and $\pi(\mathbf{R})<\infty$ then $H(u,$. is $C^{1}$ on $K \cap\{(x, y): y>0\}$. Moreover, if $u_{y}\left(x, 0^{+}\right), u_{y y}\left(x, 0^{+}\right)$exist and are finite, then so is $H\left(u,\left(x, 0^{+}\right)\right)$. 
Proof. i) We aim to prove that $P(z, t) \leq 0$ where

$$
P(z, t):=u(x, y(1+t)) I(z, t)-u(x, y)-u_{y}^{\prime}(x, y) y t
$$

It is clear that $P(z, t)=0$ in the case where $y=0$. Otherwise, since $u \geq 0$, we deduce that $P(z, t) \leq u(x, y(1+t))-u(x, y)-u_{y}^{\prime}(x, y) y t$. So,

$$
\begin{aligned}
P(z, t) & \leq u(x, y(1+t))-u(x, y)-u_{y}^{\prime}(x, y) y t, \\
& \leq\left(u_{y}^{\prime}\left(x, \theta_{x}\right)-u_{y}^{\prime}(x, y)\right) y t,
\end{aligned}
$$

where $\theta_{x} \in[y, y(1+t)]$. Since the function $y \rightarrow u(x, y)$ is concave, $y \mapsto u_{y}^{\prime}(x, y)$ is non increasing on $C^{1}\left(K \backslash \mathbf{R} e_{1}\right)$ hence $P(z, t) \leq 0$.

ii) Assume that $(x, y) \in K$ and $y>0$. Let $z_{n}:=\left(x_{n}, y_{n}\right) \in K, y_{n}>0$ be a sequence convergent to $z:=(x, y)$. Since $u$ is locally Lipschitz on $K \backslash \mathbf{R} e_{1}$, we have for $n$ large enough $P\left(\left(x_{n}, y_{n}\right), t\right)-P((x, y), t)=o(1)$ uniformly on $t \in(-1,0)$. We now consider $t \geq 0$. Using the homogeneity of $u$, we obtain

$u\left(x_{n}, y_{n}+y_{n} t\right)-u(x, y+y t)=(1+t)^{\gamma}\left(u\left(\frac{x_{n}}{1+t}, y_{n}\right)-u\left(\frac{x}{1+t}, y\right)\right)=(1+t)^{\gamma} o(1)$, uniformly on $t \geq 0$. Finally,

$$
u\left(x_{n}, y_{n}\right)-u(x, y)=o(1)
$$

and, similarly,

$$
u_{y}^{\prime}\left(x_{n}, y_{n}\right) y_{n} t-u_{y}^{\prime}(x, y) y t=o(t)
$$

We deduce that

$$
P\left(\left(x_{n}, y_{n}\right), t\right)-P((x, y), t)=o(1) \max (1,|t|) .
$$

This implies that $H\left(u, z_{n}\right) \rightarrow H(u, z)$ when $n \rightarrow \infty$. The case $y=0$ is proved similarly, using the boundedness of $\left(u_{y}\left(z_{n}\right)\right)_{n \geq 1}$ when $y_{n} \rightarrow 0$.

iii) The proof is similar than ii).

Let us also suppose the following:

\section{Condition (II):}

$$
\beta>\frac{\gamma \mu^{2}}{2 \sigma^{2}(1-\gamma)}+h^{*}(\gamma)
$$

where the function $h^{*}$ is given in Corollary 9.2. By Corollary 9.2, Condition (I) and (II) implies the existence of a Lyapunov function with higher growing order than the Bellman function and a classical supersolution to the HJB equation. Therefore, using Proposition 6.2, we deduce that the Bellman function is finite on $K$ and continuous up to the boudary $\partial K$, see Theorem 7.4. Therefore, we deduce by Theorem 5.3 that the HJB equation admits a unique concave solution in the class $C^{1}(K)$. Actually, we prove that $W \in C^{2}(K)$ in Subsection 10.1. To do it, we first formulate some well known results from the literature on the Bellman function. Recall that these results hold provided that $W$ is continuous, concave and monotone with respect to $K$. The proofs (for continuous diffusion processes, see [12] and [9]) may be extended to the processes with jumps, as we shall see. 
Theorem 10.2 The cone $K$ can be splitted into three non-empty open disjoint cones $K_{i}, i=0,1,2$, such that $K=\bar{K}_{0} \cup \bar{K}_{1} \cup \bar{K}_{2}$ with $\bar{K}_{0}=$ cone $\left\{\tilde{g}_{1}, \tilde{g}_{2}\right\}, \bar{K}_{1}=$ cone $\left\{g_{1}, \tilde{g}_{1}\right\}$ and $\bar{K}_{2}=$ cone $\left\{\tilde{g}_{2}, g_{2}\right\}$ for some vectors $\tilde{g}_{1}, \tilde{g}_{2}$. Moreover, $K_{1}$ contains cone $\left(g_{1}, e_{1}\right)$ and $K_{2}$ contains cone $\left(2 \mu \sigma^{-2}(1-\gamma)^{-1}(1+\lambda)^{-1} g_{2}+e_{2}, g_{2}\right)$. On $K_{1} \cup K_{2}, W$ is $C^{\infty}$ and is given by

$$
\begin{array}{ll}
W(z)=a_{1} u\left(p_{1} z\right), & \text { on } K_{1}, \\
W(z)=a_{2} u\left(p_{2} z\right), & \text { on } K_{2},
\end{array}
$$

where $a_{1}, a_{2}$ are some constants such that

$$
a_{1} \geq\left(\frac{\beta}{1-\gamma}\right)^{\gamma-1}, \quad a_{2} \geq\left(\frac{\beta}{1-\gamma}\right)^{\gamma-1} \frac{1}{(1+\lambda)^{\gamma}} .
$$

Proof. We adapt the proof of [9, Proposition 4.8.2]. With $\varphi(x):=a_{1} u\left(p_{1} x\right)$, we need to show that

$$
\mathcal{L}_{0} \varphi(x)+u^{*}\left(\varphi_{x}\right)+H(\varphi, x) \leq 0,
$$

for all $x \in$ cone $\left(g_{1}, e_{1}\right)$. By Lemma 10.1 (i), we have $H(\varphi, x) \leq 0$. Moreover, as in the proof of [9, Proposition 4.8.2], $\mathcal{L}_{0} \varphi(x)+u^{*}\left(\varphi_{x}\right) \leq 0$ due to the lower bound given by Lemma 3.2. This implies that $\varphi$ is a classical super solution of our HJB equation on the subset $\hat{K}=$ cone $\left(g_{1}, e_{1}\right)$. Moreover, by construction, $W=\varphi$ on $\hat{K}$. By Theorem 5.4, we deduce that $W \leq \varphi$ on $\hat{K}$. Similarly, we follow the proof of [9, Proposition 4.8.2, statement b)] using Theorem 5.4. We then define $K_{1}, K_{2}$ as the largest sectors on which (10.44) and (10.45) hold.

Lemma 10.3 If

$$
W\left(e_{1}\right)=\frac{1}{\gamma}\left(\frac{\beta}{1-\gamma}\right)^{\gamma-1},
$$

then the axis of the abscises is not the common boundary of $K_{1}$ and $K_{2}$.

Proof. We follow the proof of [9, Lemma 4.8.4 ]. Suppose the opposite. Then, the function $\psi(z)=a_{2}\left(p_{2} z\right)^{\gamma}$ coincides with $W(z)$ on the sector cone $\left(g_{2}, e_{1}\right)$. Then, we may deduce the value of $a_{2}$ using the assumption of the lemma. Hence, we deduce with $z=(x, y)$ that

$$
\mathcal{L}_{0} \psi(z)+u^{*}\left(\psi_{x}(z)\right)=a_{2}\left(p_{2} z\right)^{\gamma-1} y\left(\frac{1}{2} \sigma^{2}(\gamma-1) \frac{y}{p_{2} z}+\mu\right)+H(\psi, z) .
$$

With $z=(x, y)$ where $x, y>0$ and $x$ is fixed, the first term in the r.h.s. above admits a lower bound $c_{x} y$ provided that $y$ is sufficiently close to 0 and $c_{x}>0$ only depends on $x, a_{2}, \gamma, \mu$ and $\lambda$. On the other hand, we may write

$$
H(\psi, z)=\frac{y^{2}}{2} \int_{-1}^{M} \psi^{\prime \prime}\left(z+y \theta_{t}\right) t^{2} \pi(d t)+\int_{M}^{\infty} P(z, t) \pi(d t),
$$

where $\left|\theta_{t}\right| \leq|t|$ for all $t \geq-1$, and $P$ is defined by (10.43). We choose $M$ large enough such that $\pi([M, \infty))$ is close to 0 . Precisely, as $\psi_{y}(z)=a_{2}((1+\lambda) x+y)^{\gamma-1}$ is bounded by a constant depending on $x$ when $y$ is small enough, we deduce that $\psi(x, \cdot)$ is Lipschitz and finally

$$
\left|\int_{M}^{\infty} P(z, t) \pi(d t)\right| \leq \epsilon_{x} y
$$


where $\epsilon_{x}$ is arbitrarily small provided that we choose $M$ large enough. We fix $M$ such that $\epsilon_{x} \leq c_{x} / 3$. If $t \in(-1, M)$, the factor $\psi^{\prime \prime}\left(z+y \theta_{t}\right)|t|$ appearing in the first term of the r.h.s. of (10.46) is also bounded by a constant depending on $M$ but we may choose $y$ small enough such that $\psi^{\prime \prime}\left(z+y \theta_{t}\right)|t| y$ is as small as we want. We finally deduce that $|H(\psi, z)| \leq\left(2 c_{x} / 3\right) y$ provided that $y$ is small enough. This implies that $\mathcal{L}_{0} \psi(z)+u^{*}\left(\psi_{x}(z)\right)>0$ for some $z$ small enough. This yields a contradiction.

10.1 Reduction to one variable and regularity of the value function.

Using the homogeneity property of the Bellman function we reduce our problem to the case of one variable by considering the restriction of the Bellman function on the intersection of the line $\{\mathrm{z}=(\mathrm{x}, \mathrm{y}): \mathrm{x}+\mathrm{y}=1\}$ with the interior of $K$. Indeed, if we define $\psi(t):=W(1-t, t), t \in \Delta:=\left[-\frac{1}{\lambda}, 1+\frac{1}{\lambda}\right]$, so that we may reconstruct $W$ from $\psi$ by the formula

$$
W(x, y)=(x+y)^{\gamma} \psi\left(\frac{y}{x+y}\right), \quad(x, y) \in \operatorname{int} K .
$$

As in [12] and [9], we may show that $\psi$ is the viscosity solution to the new HJB equation obtained by the change of variables above:

$$
\max _{i=0,1,2} l_{i}(f)=0
$$

with the two first-order operators

$$
l_{1}(f):=-\lambda \gamma f+(1+\lambda z) f^{\prime}, \quad l_{2}(f):=-\lambda \gamma f-(1+\lambda-\lambda z) f^{\prime},
$$

and the second-order operator

$$
l_{0}(f):=f_{2} f^{\prime \prime}+f_{1} f^{\prime}+f_{0} f+\frac{1-\gamma}{\gamma}\left[\gamma f-z f^{\prime}\right]^{\frac{\gamma}{\gamma-1}}+H\left(z, f, f(z), f^{\prime}(z)\right),
$$

where

$$
\begin{aligned}
f_{2}(z) & :=\frac{1}{2} \sigma^{2} z^{2}(1-z)^{2} \\
f_{1}(z) & :=-\sigma^{2}(1-\gamma) z(1-z)(z-\theta), \quad \theta:=(1-\gamma)^{-1} \mu \sigma^{-2}, \\
f_{0}(z) & :=\frac{1}{2} \sigma^{2} \gamma(\gamma-1) z^{2}+\gamma \mu z-\beta, \\
H\left(z, f, v, v^{\prime}\right) & :=\int_{-1}^{\infty}\left[(1+z t)^{\gamma} f\left(\frac{z+z t}{1+z t}\right) 1_{(1-z, z(1+t)) \in K}-v-z t\left(\gamma v+(1-z) v^{\prime}\right)\right] d \pi(t) .
\end{aligned}
$$

Recall that $W$ is concave by Theorem 5.5. Then, the function $\psi$ defined above is also concave on $\Delta$ and its derivatives $\psi^{\prime}, \psi^{\prime \prime}$ exist almost everywhere. Therefore, (10.47) holds in the classical sense as stated in [4, Lemma 6.1]. Moreover, $\psi$ being concave, it admits left and right derivatives which are respectively left and right continuous and such that the inequality $D^{+} \psi \leq D^{-} \psi$ holds and is strict only on a countable set. Moreover, by Theorem $10.2, \psi(z)>0$ when $z$ is sufficiently close to $\lambda^{-1}$ or $1+\lambda^{-1}$. Since $\psi$ is concave, we deduce that $\psi>0$ on $\operatorname{int} \Delta$.

We now adapt some results from the literature about regularity of the Bellman function. We mainly focus on the extra term $H\left(z, \psi, \psi(z), \psi^{\prime}(z)\right)$. 
Proposition 10.4 The function $\psi$ is continuously differentiable on the interval int $\Delta$ except, maybe at zero. If $\psi^{\prime}$ has a discontinuity at 0 , then

$$
\psi(0)=\frac{1}{\gamma}\left(\frac{1-\gamma}{\beta}\right)^{1-\gamma}=\frac{1}{\gamma} \kappa_{*}^{1-\gamma} .
$$

Proof. We adapt the proof of [9, Lemma 4.8.6]. The only difference is due to the operator $H$. Clearly, this does not change the proof when $z \notin\{0,1\}$ as $H$ takes finite values. When $z=1$, we need to show the extra property that $H\left(z_{n}, \psi, \psi^{\prime}\right) \rightarrow$ $H\left(1, \psi, D^{ \pm} \psi\right)$ as $z_{n} \rightarrow 1$ with $z_{n}<1$ and $z_{n}>1$ respectively. To see it, observe that $1_{(1-z, z(1+t)) \in K}=1, \pi(d t)$ a.s., if $z \in[0,1]$. If the sequence $z_{n}>1$ is such that $z_{n} \rightarrow 1$, then $1_{\left(1-z_{n}, z_{n}(1+t)\right) \in K} \rightarrow 1$ if $t>-1$. Moreover, if $z$ bounded in a neighbourhood of 1 , the quantity $z(1+t) /(1+z t)$ is uniformly bounded in $t>-1$ and in $z$. As $D^{ \pm} \psi(1)$ exists, we then deduce that the integrand defining $H\left(z_{n}, \psi, \psi^{\prime}\right)$ is uniformly bounded by $c \min (1,|t|)$ for some constant $c>0$ independent of $n$. This implies that the dominated convergence theorem applies. Finally, when $z_{n} \uparrow 1$, we get that $H\left(z_{n}, \psi, \psi^{\prime}\right) \rightarrow H\left(1, \psi, D^{-} \psi\right)$. As in [9, Lemma 4.8.6], we finally get that

$$
\begin{aligned}
& f_{0}(1) \psi(1)+u^{*}\left(\gamma \psi(1)-D^{-} \psi(1)\right)+H\left(1, \psi, D^{-} \psi\right) \leq 0, \\
& f_{0}(1) \psi(1)+u^{*}\left(\gamma \psi(1)-D^{+} \psi(1)\right)+H\left(1, \psi, D^{+} \psi\right) \leq 0,
\end{aligned}
$$

where $u^{*}(x):=x^{\gamma(\gamma-1)^{-1}}$. Let $\alpha \in(0,1)$ be such that $p=\alpha D^{-} \psi+(1-\alpha) D^{+} \psi$. By (10.49) and (10.50), we deduce that

$$
f_{0}(1) \psi(1)+\alpha u^{*}\left(\gamma \psi(1)-D^{-} \psi(1)\right)+(1-\alpha) u^{*}\left(\gamma \psi(1)-D^{-} \psi(1)\right)+H(1, \psi, p) \leq 0 .
$$

Since $u^{*}$ is strictly convexe, we get that $f_{0}(1) \psi(1)+u^{*}(\gamma \psi(1)-p)+H\left(1, \psi, D^{+} \psi\right)<0$, in contradiction with [9, Lemma 4.8.6, Inequality (4.8.20]. The conclusion follows in the case where $z=1$.

In the case of $z=0$, we also need to verify the convergence of $H\left(z_{n}, \psi, \psi^{\prime}\right)$ to $H\left(0, \psi, \psi^{\prime}\right)$ as $z_{n} \rightarrow 0$ with $z_{n} \in(0,1)$. In this case, $1_{\left(1-z_{n}, z_{n}(1+t)\right) \in K}=1$ for all $n$ if $t>-1$. We verify that the quantity $z(1+t) /(1+z t)$ is uniformly bounded in $t>-1$ and $z \geq 0$. With $z>0$, we have $1+t z \geq z+t z=z(1+t)>0$ hence $0 \leq z(1+t) /(1+z t) \leq 1$. Therefore, the dominated convergence theorem also applies and we get that $f_{0}(0) \psi(0)+u^{*}(\gamma \psi(0))+H(0, \psi, 0) \leq 0$ and finally $f_{0}(0) \psi(0)+$ $u^{*}(\gamma \psi(0))+H(0, \psi, 0)=0$ if $\psi^{\prime}$ admits a discontinuity at zero. Since $H(0, \psi, 0)=0$, the conclusion follows.

A direct consequence of the proposition above is that the Bellman function is $C^{1}$ on int $K \backslash \mathbf{R}_{+} e_{1}$. More precisely we have:

Corollary 10.5 The value function is $C^{1}$ on int $K \backslash \mathbf{R}_{+} e_{1}$. If $\psi$ is not $C^{1}$ on $\mathbf{R}_{+} e_{1}$, then (10.48) holds. Furthermore, even if $\psi$ is not $C^{1}$ on $\mathbf{R}_{+} e_{1}$, the partial derivative $W_{x}$ is defined and continuous, and the one-sided derivatives $W_{y}(x, 0 \pm)$ are also defined and satisfy the one-sided continuity conditions

$$
W_{y}(x, 0 \pm)=\lim _{(\xi, \eta) \rightarrow(x, 0 \pm)} W_{y}(\xi, \eta)=x^{\gamma-1}\left(\gamma \psi(0)+D^{ \pm} \psi(0)\right), \quad x>0 .
$$

Proof. The first claim is an immediate consequence of Proposition 10.4. When $y=0$, $W(x, y)=x^{\gamma} \psi(0)$ so that $W_{x}(x, y)$ exists and is given by $W_{x}(x, y)=\gamma x^{\gamma-1} \psi(0)$. 
Otherwise, when $y \neq 0$ or equivalently $z=z(x, y)=y /(x+y) \neq 0$, then $\psi$ is differentiable at the point $z$ hence $W(x, y)=(x+y)^{\gamma} \psi(z(x, y))$ exists and is given by

$$
W_{x}(x, y)=\gamma(x+y)^{\gamma-1} \psi(z(x, y))+(x+y)^{\gamma} z_{x}(x, y) \psi^{\prime}(z(x, y)) .
$$

Since $D^{ \pm} \psi(0)$ exists, it is natural to take the convention $0 \times \psi^{\prime}(0)=0$ even if $\psi^{\prime}(0)$ does not exist. It follows that (10.51) holds everywhere. In particular, $W_{x}$ is continuous.

Similarly, when $y \neq 0$, i.e. $z(x, y) \neq 0, W_{y}(x, y)$ exists and is given by

$$
W_{y}(x, y)=\gamma(x+y)^{\gamma-1} \psi(z(x, y))+(x+y)^{\gamma} z_{y}(x, y) \psi^{\prime}(z(x, y)) \text {. }
$$

The claim follows.

Lemma 10.6 The interior of $K_{0}$ is nonempty.

Proof. It suffices to micmic the proof of [9, Lemma 4.8.7] by virtue of Proposition 10.4 and Lemma 10.3.

Since $K_{0} \neq \emptyset$ and $K_{1}$ contains cone $\left(g_{1}, e_{1}\right)$, there exists two real numbers $z_{1}, z_{2} \in$ $\Delta$ satisfying

$$
0 \leq z_{1}<z_{2}<1+\frac{1}{\lambda}
$$

such that

$$
K_{0}=\left\{(x, y) \in \operatorname{int} K: z_{1}<\frac{y}{x+y}<z_{2}\right\}
$$

Moreover, we have

$$
\begin{aligned}
& \psi(z)=\kappa_{1}(1+\lambda z)^{\gamma}, \quad z \in\left[-\lambda^{-1}, z_{1}\right] \\
& \psi(z)=\kappa_{2}(1+\lambda-\lambda z)^{\gamma}, \quad z \in\left[z_{2}, 1+\lambda^{-1}\right] .
\end{aligned}
$$

for some constants $\kappa_{i}, i=1,2$.

Proposition 10.7 The point $e_{1}$ belongs to int $K_{1}$.

Proof. We adapt the proof of [9, Proposition 4.8.8]. In the case where $\gamma W(e 1)>\kappa_{*}^{\gamma-1}$, the proof is based [9, Lemma 4.2.5.] that we need to verify for global viscosity solutions, i.e. when replacing local minimum by global minimum. As a real-valued continuous function admits a global minimum on a compact subset, we may easily adapt the proof provided that [9, Lemma 4.2.4] also holds. To see it, we adapt the proof of the latter replacing local by global domination. At last, if $\gamma W(e 1)>\kappa_{*}^{\gamma-1}$, we observe that $H\left(z_{n}, \psi, \psi^{\prime}\right) \rightarrow 0$ as $z_{n} \rightarrow 0$. This is indeed shown in the last part of the proof of Proposition 10.4. We then conclude.

Corollary 10.8 The function $\psi$ is $C^{1}$.

Proposition 10.9 We have $l_{1} \psi(z)<0$ and $l_{2} \psi(z)<0$ for all $z \in\left(z_{1}, z_{2}\right)$.

Proof. We follow the reasoning given in [12, Section 6]. Using the same notations, as $\psi$ is $C^{1}$, we deduce by [12, Proposition 6.2.] that the sub differential is a singleton, i.e. $\partial W(x, y)=\left\{W^{\prime}(x, y)\right\}$. Therefore, the functions $\theta^{+}$and $\theta^{-}$defined in [12, Section 6] 
coincide with the function $\theta^{2}:=(1+\lambda)^{-1} g_{2} W^{\prime 2}$. We parametrize the half-line $D_{2}$ originating at $\left(0, g_{1}\right)$ and parallel to $\left(0, g_{2}\right)$ as in [12], i.e.

$$
D_{2}:=\left\{(x(\rho), y(\rho)):=\left(1-\frac{\rho}{1+\lambda},-\frac{1}{1+\lambda}+\rho\right), \quad \rho \geq 0\right\} .
$$

Since $W$ is concave, [12, Property (6.6)] holds hence the function $\rho \mapsto \theta^{2}(x(\rho), y(\rho))$ is non increasing as stated by $[12$, Lemma $6.3,(6.7)]$. Let us define the coefficient ${ }^{3}$

$$
\rho_{2}:=\inf \left\{\rho: \theta^{2}(x(\rho), y(\rho))=0 .\right.
$$

Observe that $\rho_{2} \in(0, \infty)$ by Theorem 10.2. By [12, Lemma $\left.6.3,(6.8)\right]$, and by continuity, we get the property $\theta^{2}\left(x(\rho), y(\rho)=0\right.$ for all $\rho \geq \rho_{2}$ since $\theta^{2} \geq 0$ on $K$ and $\rho \mapsto \theta^{2}(x(\rho), y(\rho))$ is non increasing. Moreover, it is trivial that $\theta^{2}(x(\rho), y(\rho))>0$ for all $\rho<\rho_{2}$. This implies that the conic sector generated by the points of $D_{2}$ parametrized by $\rho \geq \rho_{2}$ coincides with $\overline{K_{2}}$ of Theorem 10.2. Similarly, We parametrize the half-line $D_{1}$ originating at the $\left(0, g_{2}\right)$ and parallel to $\left(0, g_{1}\right)$ :

$$
D_{1}=\left\{(x(\rho), y(\rho)):=\left(-\frac{1}{1+\lambda}+\rho, 1-\frac{\rho}{1+\lambda}\right), \quad \rho \geq 0\right\} .
$$

We also define $\theta^{1}:=(1+\lambda)^{-1} g_{1} W^{\prime}$ and

$$
\rho_{1}:=\inf \left\{\rho: \theta^{1}(x(\rho), y(\rho))=0 .\right.
$$

Similarly, we obtain that the conic sector generated by the points of $D_{1}$ parametrized by $\rho \geq \rho_{1}$ coincides with $\overline{K_{1}}$ of Theorem 10.2. Moreover, by definition of $\rho_{1}$, we get that $\theta^{1}(x(\rho), y(\rho))>0$ for all $\rho<\rho_{1}$. This means that $l_{1}(\psi)<0$ and $l_{2}(\psi)<0$ on $\left(z_{1}, z_{2}\right)$.

In order to apply the Itô formula and construct an optimal control, we need for the value function $W$ to be $C^{2}$ across the boundary of the cone $K_{0}$, except at 0 . To do so, we introduce the following local operator deduced from the global operator $l_{0}$ by freezing the value function $\psi$.

$$
\bar{l}_{0}(f):=f_{2} f^{\prime \prime}+f_{1} f^{\prime}+f_{0} f+\frac{1-\gamma}{\gamma}\left[\gamma f-z f^{\prime}\right]^{\frac{\gamma}{\gamma-1}}+\bar{H}\left(z, f(z), f^{\prime}(z)\right),
$$

where $\bar{H}\left(z, v, v^{\prime}\right)=H\left(z, \psi, v, v^{\prime}\right)$. Although, by doing so, $\psi$ is no more a viscosity solution of the (new) local operator but it is only a viscosity solution in a weak sens we precise:

Definition 10.10 A function $v \in C(K)$ is a weak viscosity supersolution (resp. subsolution) of (4.12) on a subset $\tilde{K} \subseteq K$ if and only if, for every point $x \in$ int $\tilde{K}$, the inequality $\mathcal{L} f(x) \leq 0$ (resp. $\mathcal{L} f(x) \geq 0$ ) holds for any function $\phi \in C^{2}(x)$ satisfying $(v-\phi)(x)<0$ (resp. $(v-\phi)(x)>0)$ such that the difference $v-\phi$ attains its global minimum (resp. maximum) on $K$ at $x$.

As usual, weak viscosity solution is both a weak viscosity supersolution and weak viscosity subsolution. Adapting the proof of [9, Lemma 4.2.5], we get the following:

\footnotetext{
2 Note that the coefficient $(1+\lambda)$ in our paper corresponds to the coefficient $(1-\lambda)^{-1}=$ $(1-\mu)^{-1}$ of [12].

3 Denoted by $\rho_{0}$ in [12].
} 
Lemma 10.11 Let $\psi \in C^{1}(a, b)$ be a weak viscosity solution of the (local) equation

$$
\psi^{\prime \prime}(z)=G\left(\psi^{\prime}(z), \psi(z), z\right)
$$

on a nonempty subinterval $\left(a^{\prime}, b^{\prime}\right) \subseteq(a, b)$. Suppose that $z \mapsto G\left(\psi^{\prime}(z), \psi(z), z\right)$ is continuous on $\left[a^{\prime}, b^{\prime}\right]$. Then, $\psi \in C^{2}\left(a^{\prime}, b^{\prime}\right)$ and the equation holds in the classical sense.

Proof. It suffices to repeat the proof of [9, Lemma 4.2.5]. Indeed, with the same notations, the case of interest is when the minimum of $\psi-\psi_{\epsilon}$ is negative. In that case, we use the weak supersolution property. Otherwise, $\psi-\psi_{\epsilon} \geq 0$, i.e. $\psi \geq \psi_{\epsilon}$ on $\left[z_{1}, z_{2}\right]$, which is the desired conclusion. Symmetrically, when the maximum of $\psi-\psi_{\epsilon}$ is positive, we use the weak subsolution property. On the contrary, we have $\psi \leq \psi_{\epsilon}$ and the conclusion follows.

Proposition 10.12 Suppose that $l_{1} \psi\left(z_{0}\right)<0$ and $l_{2} \psi\left(z_{0}\right)<0$ at some point $z_{0} \in$ $\left(0,1+\lambda^{-1}\right] \backslash\{1\}$. Then, if $\pi(\mathbf{R})<\infty, \psi \in C^{2}\left(z_{0}\right)$.

Proof. By continuity, we may assume that $l_{1} \psi(z)<0$ and $l_{2} \psi(z)<0$ for all $z$ in some interval $[a, b]$ where $a<z_{0}<b$. As $z_{0} \neq 1$, we may suppose that $1 \notin[a, b]$. We show that $\psi$ is a weak viscosity solution to the equation $\bar{l}_{0} \psi=0$ on $[a, b]$.

First, as $\psi$ is a viscosity supersolution to the equation $\max _{i=0,1,2} l_{i} \psi=0$, this is also a viscosity supersolution to $l_{0} \psi=0$. Indeed, $\max _{i=0,1,2} l_{i} \phi \leq 0$ implies $l_{0} \phi \leq 0$ for any test function $\phi$.

Let us now consider any function $f \in C^{2}\left(z_{0}\right)$ where $z_{0} \in(a, b)$ and suppose that that the difference $\psi-f$ attains its global minimum on $K$ at $z_{0}$ such that $(\psi-f)\left(z_{0}\right)<$ 0 . Let us consider $\tilde{f}^{r}:=f \xi^{r}+\psi\left(1-\xi^{r}\right)$ where, by the one dimensional version of Lemma 11.1, $\xi^{r} \in[0,1]$ is infinitely differentiable, vanishes outside the ball of center $z_{0}$ and radius $r \rightarrow 0$ and $\xi^{r}=1$ on a smaller ball around $z_{0}$. Note that $\psi-\tilde{f}^{r}=(\psi-f) \xi^{r}$. Since $\xi^{r} \geq 0,\left(\psi-\tilde{f}^{r}\right)(z) \geq(\psi-f)\left(z_{0}\right) \xi^{r}(z)$. As $(\psi-f)\left(z_{0}\right)<0$ and $\xi^{r} \leq 1$, $(\psi-f)\left(z_{0}\right) \xi^{r}(z) \geq(\psi-f)\left(z_{0}\right)$. This implies that $\left(\psi-\tilde{f}^{r}\right)(z) \geq\left(\psi-\tilde{f}^{r}\right)\left(z_{0}\right)$, i.e. $\psi-\tilde{f}^{r}$ admits a global minimum at $z_{0}$. Therefore, $l_{0} \tilde{f}^{r}\left(z_{0}\right) \leq 0$. Notice that by assumption $f\left(z_{0}\right)>\psi\left(z_{0}\right)$ so that, by continuity of $f$, we may choose $r \rightarrow 0$ and $\epsilon>0$ small enough such that $\tilde{f}^{r}(z) \geq \psi(z)-\epsilon h(z)$ on $z \in D_{h}$ where $h$ is a continuous function we may choose arbitrarily. Actually, we choose $h$ on a set $D_{h} \ni z_{0}$ such that $h\left(z_{0}\right)=1$. Precisely, if $z_{0}>1, h$ is defined on $D_{h}=[1, \infty)$ with $h=0$ on $\left[1,\left(z_{0}+1\right) / 2\right]$. If $z_{0}<1$, $h$ is defined on $D_{h}=[0,1]$ and $h=0$ on $\left[\left(z_{0}+1\right) / 2,1\right]$. Notice that the range of the mapping $\delta: t \mapsto\left(z_{0}+z_{0} t\right) /\left(1+z_{0} t\right)$ is $D_{h}$ when $1+z_{0} t>0$ and $\lim _{t \rightarrow \infty} \delta(t)=1$. Therefore, the integral

$$
\int_{-1}^{\infty}\left(1+z_{0} t\right)^{\gamma} h\left(\frac{z_{0}+z_{0} t}{1+z_{0} t}\right) 1_{\left(1-z_{0}, z_{0}(1+t)\right) \in K} d \pi(t)
$$

is well defined and finite as $h\left[\left(z_{0}+z_{0} t\right) /\left(1+z_{0} t\right)\right]$ vanishes when $\left(z_{0}+z_{0} t\right) /\left(1+z_{0} t\right)$ is closed to 1 , i.e. when $t$ is closed to $\infty$. From the inequalities $l_{0} \tilde{f}^{r}\left(z_{0}\right) \leq 0$ and $\tilde{f} \geq \psi-\epsilon h$ on $z \in D_{h}$, we then deduce that $\bar{l}_{0} f\left(z_{0}\right) \leq 0$ as $\epsilon \rightarrow 0$, i.e. we have replaced $\tilde{f}$ by $\psi$ in the global operator.

Since $\psi$ is a viscosity subsolution of the equation $\max _{i=0,1,2} l_{i} \psi=0$, for any test function dominating $\psi$, we have $\max _{i=0,1,2} l_{i} \phi \geq 0$. Moreover, $\max _{i=1,2} l_{i} \phi=$ $\max _{i=1,2} l_{i} \psi<0$ since $l_{1}$ and $l_{2}$ are local operators only depending on the values of $\psi$ and its first derivative at the considered point $z \in(a, b)$. It follows that $l_{0} \phi=$ 
$\max _{i=0,1,2} l_{i} \phi \geq 0$. As above, we deduce that $\psi$ is a weak viscosity subsolution of the equation $\bar{l}_{0} \psi=0$ on $[a, b]$ hence $\psi$ is finally a weak viscosity solution of the equation $\bar{l}_{0} \psi=0$ on $[a, b]$.

Let us define the local operator $\zeta(f, z):=\bar{l}_{0} f(z)-f_{2}(z) f^{\prime \prime}(z)$. Since $\pi(\mathbf{R})<\infty$, we deduce by Corollary 10.8 and Lemma 10.1 (ii) that $z \mapsto \zeta(\psi, z)$ is continuous on $\left(0,1+\lambda^{-1}\right]$. We deduce that $z \mapsto G\left(\psi^{\prime}(z), \psi(z), z\right):=-\zeta(f, z) / f_{2}(z)$ is continuous. Recall that the operator which appears in the definition of $G$ is local as we have frozen the dependence in the test function $\psi$ before. Therefore, by the weak solution property and Lemma 10.11, $\psi \in C^{2}[a, b]$.

The following result corresponds to [12, Proposition 8.5]. Its proof may be easily adapted to the case with jumps. To do so, we use the continuity of the function $z \rightarrow$ $H\left(z, \psi, \psi^{\prime}\right)$ as stated by Lemma $\left.10.1 \mathrm{i}\right)$.

Proposition 10.13 Suppose that $\pi(\mathbf{R})<\infty$. The function $\psi$ is $C^{2}$ on the set $\left(z_{1}, z_{2}\right) \backslash$ $\{1\}$ and satisfies the HJB equation $\psi=0$ on this set in the classical sense.

The proposition above implies that the value function satisfies the HJB equation $\mathcal{L}_{0}(W)+U^{*}\left(W_{x}\right)=0$ on $K_{0} \backslash \mathbf{R} e_{2}$ in the classical sense and it is $C^{2}$ on this set. It remains to study $W$ on the set $\mathbf{R} e_{2}$. To do so, we follow the proof of in [12, Theorem 9.1].

Proposition 10.14 The second derivative $W_{y y}$ is well defined and is continuous accross $\mathbf{R}_{+} e_{2}$. Moreover, $W$ satisfies the equation $\mathcal{L}_{0}(W)+U^{*}\left(W_{x}\right)=0$ on $K_{0}$ in the classical sense.

Finally, we deduce the following:

Corollary 10.15 Suppose that $\pi(\mathbf{R})<\infty$. Then, the value function $W$ is $C^{2}$.

\subsection{Optimal control}

The following important result provides an optimal policy for the optimization problem. The proof is based on the resolution of a Skorohod problem described in Appendix.

Theorem 10.16 Suppose that the boundaries of $K_{0}$ are different from the $x$ - and $y$ axes. Let $(x, y) \in \bar{K}_{0}$, then the Skorokhod problem (11.62) with $\sigma\left(V_{t}\right):=\left(-W_{x}\left(V_{t}\right)^{\frac{1}{\gamma-1}}, V_{t}\right)$ admits a unique solution. Moreover, the portfolio process $V$ participating in the solution of this problem is an optimal portfolio. An optimal strategy is given by the formula

$$
\begin{aligned}
B_{t} & =\int_{0}^{t} g\left(V_{s}\right) d k_{s}, \\
c_{t} & =W_{x}^{\frac{1}{\gamma-1}},
\end{aligned}
$$

where $W$ is the Bellman function.

Proof. Existence of a solution to the Skorohod problem holds by Theorem 11.4. Note that $\theta^{\pi}=\infty$ since $V_{t}^{+} \in K_{0}, \forall t$, and $W_{x}$ is positive (hence (10.55) makes sense). We shall only consider the case where $K_{0}$ is included in the first quadrant. Otherwise, we refer to Remark 10.18. By Propositions 10.9 and 10.13, the function $\psi$ coincides on the interval $\left(z_{1}, z_{2}\right)$ with a $C^{2}$-function $\tilde{\psi}$ defined on $\left(-\lambda^{-1}, 1+\lambda^{-1}\right)$. Indeed, it suffices to replace $\psi$ by suitable parabolic functions outside $\left(z_{1}, z_{2}\right)$. In particular, since 
$\tilde{\psi}^{\prime}$ is Lipschitz on $\left[z_{1}, z_{2}\right]$ and $W(z)=(x+y)^{\gamma} \tilde{\psi}(z)$ where $z=y /(x+y)$ on $K_{0}$, we also deduce that $W^{\prime}$ is also Lipschitz on $K_{0}$. Moreover, by [9, Lemma 4.7.1, Corollary 4.7.6], $W \neq 0$ implies that $W^{\prime}(z) \in K^{*} \backslash\{0\} \subseteq(0, \infty)^{2}$ hence $W_{x}^{\prime}(z)>0$ for all $z$. It follows that $\sigma$ is locally Lipschitz on the set $K_{0}$. We then deduce that the Skorokhod problem admits a unique solution. We check the second assertion. Applying Lemma 4.8 , since the term $R$ of the expansion is negative, we have

$$
\begin{aligned}
e^{-\beta t} W\left(V_{t}^{+}\right)+J_{t}^{\pi} & \leq W(x, y)+N_{t} \\
& +\int_{0}^{t} e^{-\beta u}\left[\mathcal{L}_{0} W\left(V_{u_{-}}\right)-W^{\prime}\left(V_{u_{-}}\right) c_{u}+U\left(c_{u}\right)\right] d u
\end{aligned}
$$

Moreover, when $c$ is defined by (10.55), we have $W_{x}\left(V_{u_{-}}\right) c_{u}+U\left(c_{u}\right)=U^{*}\left(c_{u}\right)$. Therefore, the last integral term in the equality above is zero by virtue of Proposition 10.14. It remains to prove that $N$ is a martingale and

$$
\lim _{n \rightarrow \infty} \mathbb{E} e^{-\beta t_{n}} W\left(V_{t_{n}}^{+}\right) \rightarrow 0,
$$

for a sequence of real numbers $t_{n} \rightarrow \infty$. To prove (10.56), we observe that $|W(z)| \leq$ $\kappa|z|^{\gamma}$ and $\left|W_{x}(z)\right| \leq \kappa|z|^{\gamma-1}$ where $\kappa$ is an upper bound of $W$ and $W_{x}$ on the intersection $\Delta_{0}$ of the set $K_{0}$ with the line $x+y=1$. This is deduced from the continuity of $W$ on $\Delta_{0}$ and the fact that $\psi^{\prime}(0+)<\infty$. We deduce the existence of a constant $\kappa$ such that

$\mathbb{E} \int_{0}^{\infty} e^{-\beta t} W\left(V_{t}^{+}\right) d t \leq \kappa \mathbb{E} \int_{0}^{\infty} e^{-\beta t}\left|V_{t}^{+}\right|^{\gamma} d t \leq \kappa \mathbb{E} \int_{0}^{\infty} e^{-\beta t} u\left(c_{t}\right) d t \leq \kappa W(x)$.

Since $W$ is finite, this implies the existence of a sequence $t_{n} \uparrow \infty$ such that (10.56) holds. Details of this assertion are given in Lemma 10.17. We now prove that $N_{t}$ is a true martingale. Indeed, by a similar argument, we have

$$
\left|z W_{y}(z)\right| \leq \kappa|z|^{\gamma} \leq(1+|z|), \quad z \in K_{0} .
$$

Hence, we infer that the stochastic process $\int_{0}^{\cdot} e^{-\beta u} W_{y}\left(V_{u_{-}}^{2}\right) V_{u-}^{2} \sigma d W_{u}$ is a martingale. The second process defining $N$ is the integral with respect to the Lévy measure. Observe that, for each fixed $s$, we have $I\left(V_{s_{-}}, z\right)=1$ (because $\left.V_{s}^{+} \in \bar{K}_{0}, \forall s\right)$. Moreover, using the finite Taylor expansion, we get

$$
\left|W\left(\widetilde{V}_{s_{-}}+\operatorname{diag}\left(\widetilde{V}_{s_{-}}\right) z\right)-W\left(\widetilde{V}_{s_{-}}\right)\right| \leq\left|W^{\prime}(\eta)\right|\left|\operatorname{diag}\left(\widetilde{V}_{s_{-}}\right) z\right|
$$

where $\eta \in\left[\widetilde{V}_{s_{-}}, \widetilde{V}_{s_{-}}+\operatorname{diag}\left(\widetilde{V}_{s_{-}}\right) z\right]$ satisfies $|\eta| \leq\left|\widetilde{V}_{s_{-}}\right|(1+|z|)$. It follows that

$$
\left|W\left(\widetilde{V}_{s_{-}}+\operatorname{diag}\left(\widetilde{V}_{s_{-}}\right) z\right)-W\left(\widetilde{V}_{s_{-}}\right)\right| \leq \kappa|\eta|^{\gamma-1}\left|\operatorname{diag}\left(\widetilde{V}_{s_{-}}\right) z\right| \leq \kappa\left|\widetilde{V}_{s_{-}}\right|^{\gamma}(1+|z|)^{\gamma-1}|z|,
$$

where the last inequality is deduced from the inequality $\left|W^{\prime}(\eta)\right| \leq \kappa|\eta|^{\gamma-1}$. We then obtain that

$$
\left|W\left(\widetilde{V}_{s_{-}}+\operatorname{diag}\left(\widetilde{V}_{s_{-}}\right) z\right)-W\left(\widetilde{V}_{s_{-}}\right)\right| \leq \kappa\left|\widetilde{V}_{s_{-}}\right|^{\gamma}|z| .
$$

Therefore, as the Lévy process is of finite activity and (10.57) holds,

$\mathbb{E} \int_{0}^{t} \int_{\mathbf{R}} e^{-\beta u}\left|W\left(\widetilde{V}_{s_{-}}+\operatorname{diag}\left(\widetilde{V}_{s_{-}}\right) z\right) I\left(\widetilde{V}_{s_{-}}, z\right)-W\left(\widetilde{V}_{s_{-}}\right)\right| \pi(d z) d s \leq \kappa W(x)<\infty$. 
By [8, Theorem I.1.33 b., p73], we deduce that the purely discontinuous local martingale $N$ satisfies $\mathbb{E} \operatorname{var}(N)_{\infty}<\infty$ hence is a martingale.

To complete the proof of the theorem, we need the following lemma

Lemma 10.17 Suppose that $\int_{0}^{\infty} X_{u} d u<\infty$ where $X_{u}=\mathbb{E} e^{-\beta u} W\left(V_{u+}\right) \geq 0$. Then $\lim _{t \rightarrow \infty} X_{t} \rightarrow 0$.

Proof. Observe that $e^{-\beta t} W\left(V_{t}^{+}\right)+J_{t}^{\pi}=W(x)+N_{t}$. With $Y_{u}=e^{-\beta u} W\left(V_{u+}\right)$, we get that for $u \geq s, Y_{u}-Y_{s}=N_{u}-N_{s}-\left(J_{u}^{\pi}-J_{s}^{\pi}\right)$ where $J_{u}^{\pi}-J_{s}^{\pi} \geq 0$. Since $N$ is a martingale, we deduce that $X_{u}-X_{s} \leq 0$, i.e. $X$ is decreasing. Therefore, the integrablity of $\int_{0}^{\infty} X_{u} d u$ ensures that $\lim _{t \rightarrow \infty} X_{t} \rightarrow 0$. Indeed, if $\lim _{t \rightarrow \infty} X_{t} \rightarrow c>0$, then $\int_{0}^{\infty} X_{u} d u \geq \int_{0}^{\infty} c d u=\infty$, hence a contradiction.

Remark 10.18 The situations where $x \in K_{i}, i=1,2$, are easily reduced to the one treated in the theorem above. Indeed, recall that the function $W$ restricted on the set $K_{i}$ is constant along the direction $g_{i}, i=1,2$. Instead of considering the initial position $x \in$ $K_{i}, i=1,2$, we consider the point $\tilde{x}$ lying on the boundary of $K_{0}$ by projecting $x$ onto $K_{0}$ parallel to $g_{i}$. This translation does not change the value of the Bellman function, meaning that $W(x)=W(\tilde{x})$. Therefore, the optimal strategy for $x$ is constructed simply by adding the initial jump $\Delta B_{0}:=\tilde{x}-x$ to the optimal strategy given by the Skorokhod problem with the initial point $\tilde{x}$.

\section{Appendix: the Skorokhod problem}

11.1 Skorokhod problem for continuous diffusion processes

The construction of the optimal control for the two-dimensional optimal consumption problem we consider in Section 10 is based on the resolution of the so-called Skorokhod problem. This problem is about existence and uniqueness of the solution to a S.D.E. with reflection. We first recall some known results for the continuous diffusion case. We provide the proof for the sake of completeness. In the next section, we extend these results to finite activity pure-jumps Lévy processes.

Let $\gamma: \partial K_{0} \mapsto \mathbf{R}^{2}$ be a vector-valued function with $g(x)=-g_{i}$ on $\left(\partial K_{0} \cap \partial K_{i}\right) \backslash\{0\}$ and $\gamma(0)=0$. Let $Y$ be the process $Y_{t}=\left(Y_{0}^{1}, Y_{0}^{2}\right)+\left(t, W_{t}\right), t \geq 0$, where $W$ is a standard Brownian motion. Let $\sigma=\mathbf{R}^{2} \mapsto \mathbf{R}^{2} \times \mathbf{R}^{2}$ be a matrix-valued function which is Lipschitz-continuous.

On the closed cone $\bar{K}_{0}$ of Section 10, we consider the Skorokhod problem formulated as follows: find a pair of adapted continuous processes $V \in \mathbf{R}^{2}$ and $k \in \mathbf{R}$, starting respectively from $x \in \bar{K}_{0}$ and zero, such that $k$ is non decreasing and

$$
\begin{aligned}
& d V_{t}=\sigma\left(V_{t}\right) d Y_{t}+\gamma\left(V_{t}\right) d k_{t}, \\
& d k_{t}=I_{V_{t} \in \partial K_{0}} d k_{t}, \\
& V_{t} \in K_{0}, \forall t \geq 0 .
\end{aligned}
$$

For any compact subset $C$ of $\mathbf{R}^{2}$ and for all $\epsilon>0$, let us define

$$
C_{\epsilon}:=\bigcup_{c \in C} B(c, \epsilon)
$$

where $B(c, \epsilon)$ is the closed ball of radius $\epsilon$ and center $c$. Recall the well known result: 
Lemma 11.1 For any compact subset $C$ of $\mathbf{R}^{2}$ and for all $\epsilon>0$, there exists $\chi^{\epsilon} \epsilon$ $C^{\infty}\left(\mathbf{R}^{2},[0,1]\right)$ such that $\chi^{\epsilon}=1$ on $C_{\epsilon}$ and $\chi^{\epsilon}$ vanishes outside $C_{2 \epsilon}$.

The aim of this section is to show that the R.S.D.E (11.58) admits a solution on the set $K_{0}$ which is trapped at zero. To do so, we shall prove several intermediate lemmas. The main proof is based on the existence of a solution to a R.S.D.E. on a bounded domain $G$ if the direction of the reflection is given by a function $\gamma \in C^{2}$ satisfying the following condition (see [5]):

C1: $\gamma \in C^{2}\left(\mathbf{R}^{2}, \mathbf{R}^{2}\right)$ and there is $b \in(0,1)$ such that

$$
\bigcup_{0 \leq t \leq b} B(x-t \gamma(x), t b) \subseteq G^{c}=\mathbf{R}^{2} \backslash G, \quad \text { for all } x \in \partial G .
$$

Theorem 11.2 The Skorokhod problem (11.58) admits a solution which is trapped at zero.

Proof. Let us introduce the polygons

$$
K_{0}^{n}:=K_{0} \cap\left\{(x, y): \epsilon_{n}^{-1} \leq x+d y \leq \epsilon_{n}\right\},
$$

where $\epsilon_{n} \rightarrow \infty$. Let $D$ be the bisector of the cone $K_{0}^{n}$ and $d>0$ such that $D:=$ $\{(x, y):-d x+y=0\}$. Let $x \in \partial K_{0}^{n}$ be a starting point. The case $x=0$ being trivial, we assume that $x \neq 0$ hence $x \in K_{0}^{n}$ if $n$ is large enough.

- Step 1. There exists closed regions $\tilde{K}_{0}^{n}$ such that $K_{0}^{n} \subseteq \tilde{K}_{0}^{n} \subseteq K_{0}^{n+1}$ which satisfy Condition $\mathbf{C 1}$ for some reflection function $\gamma_{n}$ such that $\gamma_{n}(x) \rightarrow \gamma(x)$ for all $x \in \partial K_{0}$. Indeed, we denote by $a_{n}$ and $b_{n}$ the two points of $\partial K_{0} \cap\left\{(x, y): \epsilon_{n}=x+d y\right\}$ such that $y_{a_{n}}>y_{b_{n}}$. Observe that $b_{n}$ is the symmetric of $a_{n}$ with respect to the bisector $D$. Similarly, $c_{n}$ and $d_{n}$ are the two symmetric points of $\partial K_{0} \cap\left\{(x, y): \epsilon_{n}^{-1}=x+d y\right\}$. We then define $\tilde{K}_{0}^{n}$ as the polygon

$$
\tilde{K}_{0}^{n}:=K_{0} \cap\left\{(x, y):\left(\epsilon_{n}^{-1}+\epsilon_{n+1}^{-1}\right) / 2 \leq x+d y \leq\left(\epsilon_{n}+\epsilon_{n+1}\right) / 2\right\},
$$

and denote by $\tilde{a}_{n}$ and $\tilde{b}_{n}$ the two points of $\partial K_{0} \cap\left\{(x, y):\left(\epsilon_{n}+\epsilon_{n+1}\right) / 2=x+d y\right\}$ such that $y_{\tilde{a}_{n}}>y_{\tilde{b}_{n}}$. Similarly, $\tilde{c}_{n}$ and $\tilde{d}_{n}$ are the two points of $\partial K_{0} \cap\left\{(x, y):\left(\epsilon_{n}^{-1}+\right.\right.$ $\left.\left.\epsilon_{n+1}^{-1}\right) / 2=x+d y\right\}$ such that $y_{\tilde{c}_{n}}>y_{\tilde{d}_{n}}$.

Let $\eta_{1}$ be the outward normal to $\partial K_{0} \cap K_{1}$ and $\eta_{2}$ be the outward normal to $\partial K_{0} \cap K_{2}$. We consider a unit vector $g_{3}$ such that $g_{3} \eta_{1}>0$ and $g_{3}(1, d)>0$. Similarly, we define $g_{4}$ as a unit vector such that $g_{4} \eta_{2}>0$ and $g_{4}(1, d)>0, g_{5}$ is a unit vector such that $g_{5} \eta_{1}>0$ and $g_{5} \eta_{2}>0$. Let us introduce the smooth function $\gamma_{n}(x)=$ $\left(\gamma_{n}^{1}(x), \gamma_{n}^{1}(x)\right)$ with

$$
\begin{aligned}
& \gamma_{n}^{1}(x):=-\left(g_{1} \chi^{1}(x)+g_{2} \chi^{2}(x)+g_{3}\left(1-\chi^{1}(x)\right)\left(1-\chi^{4}(x)\right) \chi^{3}(x)\right) \\
& \gamma_{n}^{2}(x):=-\left(g_{4}\left(1-\chi^{2}(x)\right)\left(1-\chi^{3}(x)\right) \chi^{4}(x)+g_{5}\left(1-\chi^{1}(x)\right)\left(1-\chi^{2}(x)\right) \chi^{5}(x)\right) .
\end{aligned}
$$

By Lemma 11.1, $\chi_{i} \in C^{\infty}\left(\mathbf{R}^{2},[0,1]\right)$ for all $i=1, \cdots, 5$, Moreover, with the notation given by (11.61) above, if $\gamma_{n}$ is sufficiently closed to 0 , these functions satisfy :

$\chi^{1}(x)=1$ on $\left[d_{n}, b_{n}\right]_{\gamma_{n}}$ and $\chi^{1}(x)=0$ on $\mathbf{R}^{2} \backslash\left[d_{n}, b_{n}\right]_{2 \gamma_{n}}$,

$\chi^{2}(x)=1$ on $\left[c_{n}, a_{n}\right]_{\gamma_{n}}$ and $\chi^{2}(x)=0$ on $\mathbf{R}^{2} \backslash\left[c_{n}, a_{n}\right]_{2 \gamma_{n}}$,

$\chi^{3}(x)=1$ on $\left(C_{3}^{n}\right)_{\gamma_{n}}:=\left(\left[\tilde{e}_{n}, \tilde{b}_{n}\right] \cup\left[\tilde{b}_{n}, b_{n}\right]\right)_{\gamma_{n}}$ and $\chi^{3}(x)=0$ on $\mathbf{R}^{2} \backslash\left(C_{3}^{n}\right)_{2 \gamma_{n}}$, 
$\chi^{4}(x)=1$ on $\left(C_{4}^{n}\right)_{\gamma_{n}}:=\left(\left[\tilde{e}_{n}, \tilde{a}_{n}\right] \cup\left[\tilde{a}_{n}, a_{n}\right]\right)_{\gamma_{n}}$ and $\chi^{4}(x)=0$ on $\mathbf{R}^{2} \backslash\left(C_{4}^{n}\right)_{2 \gamma_{n}}$. $\chi^{5}(x)=1$ on $\left(C_{5}^{n}\right)_{\gamma_{n}}:=\left(\left[c_{n}, \tilde{c}_{n}\right] \cup\left[\tilde{c}_{n}, \tilde{d}_{n}\right] \cup\left[\tilde{d}_{n}, d_{n}\right]\right)_{\gamma_{n}}$ and $\chi^{5}(x)=0$ on $\mathbf{R}^{2} \backslash\left(C_{5}^{n}\right)_{2 \gamma_{n}}$.

Let us denote by $\eta(x)$ the outward normal at each point of $\partial \tilde{K}_{0}^{n}$. The mapping $\eta: \partial \tilde{K}_{0}^{n} \mapsto \mathbf{R}^{2}$ is continuous except at the points $\tilde{a}_{n}, \tilde{b}_{n}, \tilde{c}_{n}, \tilde{d}_{n}$ where it admits left and righ limits we denote by $\eta(x \pm)$. Moreover, by construction we have $\gamma_{n}(x) \eta(x-)>0$ and $\gamma_{n}(x) \eta(x+)>0$ for all $x \in \partial \tilde{K}_{0}^{n}$.

Observe that

$$
d\left(x-t \gamma_{n}(x), \partial \tilde{K}_{0}^{n}\right) \geq \min _{x \in \partial \tilde{K}_{0}^{n}} d\left(x-t \gamma_{n}(x), \partial \tilde{K}_{0}^{n}\right):=m^{n}, \quad \forall x \in \partial \tilde{K}_{0}^{n} .
$$

By a compactness argument, $m^{n}=d\left(x_{\infty}-t \gamma_{n}\left(x_{\infty}\right), \partial \tilde{K}_{0}^{n}\right)$ for some $x_{\infty} \in \tilde{K}_{0}^{n}$. Since

$$
d\left(x_{\infty}-t \gamma_{n}\left(x_{\infty}\right), \partial \tilde{K}_{0}^{n}\right) \geq-t \gamma_{n}\left(x_{\infty}\right) \eta\left(x_{\infty} \pm\right)=2 b t,
$$

where $2 b:=-\gamma_{n}\left(x_{\infty}\right) \eta\left(x_{\infty} \pm\right)>0$, we finally deduce that Condition C1 holds.

- Step 2. By virtue of [5, Corollary 5.2], since $\sigma$ is globally Lipschitz on the bounded domain $\tilde{K}_{0}^{n}$, there exists a unique strong solution $\left(V^{n}, k^{n}\right)$, starting from $x$, to the reflected S.D.E. (11.58) on the domain $\tilde{K}_{0}^{n}$. Let us introduce

$$
\begin{gathered}
\tau^{n}:=\inf \left\{t: V_{t}^{n}(1, d)=\epsilon_{n}^{-1}\right\}, \\
\rho^{n}:=\inf \left\{t: V_{t}^{n}(1, d)=\epsilon_{n}\right\},
\end{gathered}
$$

and $\mu^{n}:=\tau^{n} \wedge \rho^{n}$. On the intervall $\left[0, \mu^{n}\right]$, the process $\left(V^{n}, k^{n}\right)$ is solution to (11.58) on the domain $\tilde{K}_{0}^{n+1}$ with respect to $\gamma^{n+1}$. Indeed, on the intervall $\left[0, \mu^{n}\right]$, the reflection only occurs on the boundary $\partial K_{0}$ on which $\gamma^{n+1}$ and $\gamma^{n}$ coincides with $\gamma$. By the uniqueness property given by [5, Corollary 5.2], we deduce that $\left(V^{n}, k^{n}\right)=$ $\left(V^{n+1}, k^{n+1}\right)$ on $\left[0, \mu^{n}\right]$. It follows that $\mu^{n} \leq \mu^{n+1}$. The rest of the proof is done as in [9, page 229].

\subsection{Skorokhod problem for pure-Jumps Lévy processes}

The setting of this subsection is given in Subsection 11.1. Let $\gamma: \partial K_{0} \mapsto \mathbf{R}^{2}$ be a vector-valued function with $g(x)=-g_{i}$ on $\left(\partial K_{0} \cap \partial K_{i}\right) \backslash\{0\}$ and $\gamma(0)=0$. Recall that, as the Lévy process we consider is of finite activity, it can be represented as the sum of a compound poison process and a Wiener process. So, consider a process $Y$ defined by

$$
Y_{t}=\left(Y_{0}^{1}, Y_{0}^{2}, 0\right)+\left(t, W_{t}, N_{t}\right), \quad t \geq 0,
$$

where $W$ is a standard Brownian motion and $N$ is a pure jump process of finite activity. This means that

$$
N_{t}=\sum_{k=1}^{\tilde{N}_{t}} \Delta N_{T_{k}},
$$

where $\Delta N_{T_{k}}$ are i.i.d. random variables and $\tilde{N}_{t}=\sum_{k} 1_{T_{k} \leq t}$ is a Poisson process with jump stopping times $\left(T_{k}\right)_{k \geq 1}$. Let $\sigma=\mathbf{R}^{2} \mapsto \mathbf{R}^{2} \times \mathbf{R}^{2} \times \mathbf{R}^{2}$ be a matrix-valued function which is Lipschitz-continuous.

We consider the Skorokhod problem on $K_{0}$ formulated as follows: find a pair of adapted làdlàg (resp. càglàd) processe $V$, starting from $x \in K_{0}$ and real-valued process $k$, starting at zero and increasing, such that 


$$
\begin{aligned}
d V_{t} & =\sigma\left(V_{t-}\right) d Y_{t}+\gamma\left(V_{t}\right) d k_{t}, \\
d k_{t} & =I_{V_{t}} \in K \backslash \operatorname{int} K_{0} d k_{t}, \\
V_{t}^{+} & \in \bar{K}_{0}, \forall t \geq 0 .
\end{aligned}
$$

The goal is to show that this R.S.D.E has a solution on the set $\bar{K}_{0}$. To do so, we shall use the following:

Lemma 11.3 [Projection onto $K_{0}$ parallel to $-K$ ]

Assume that $K \subseteq \mathbf{R}^{2}$ is a constant cone satisfying the hypothesis of the introduction and $K_{0} \subseteq K$ is a closed cone with $\partial K_{0} \subseteq \operatorname{int} K$ and int $K_{0} \neq \emptyset$. For every $x \in K$, there exists a unique $y:=\mathbb{P}_{K_{0}}^{-K}(x) \in K_{0}$ such that

$$
\|x-y\|=\min _{k \in K}\{\|x-k\|: x-k \in K\} .
$$

We omit the proof which is standard. It is easily observable that the direction of $x-\mathbb{P}_{K_{0}}^{-K}(x)$ is given by $g_{2}$ if $x \in K_{2}$ and $g_{1}$ if $x \in K_{1}$.

Theorem 11.4 There exists a unique solution to the Skorokhod problem (11.62).

Proof. Let $\left(T_{k}\right)_{k \geq 1}$ be the jump stopping times of the process $Y$. Assume that we have already constructed a solution $(V, k)$ to $(11.62)$ on the interval $\left[0, T_{k}\right)$. Define

$$
V_{T^{k}}:=V_{T^{k}-}+\sigma\left(V_{T^{k}-}\right) \Delta Y_{T^{k}} .
$$

Let us introduce

$$
V_{T^{k}+}=\mathbb{P}_{K_{0}}^{-K}\left(V_{T^{k}}\right) \in L^{0}\left(\mathbf{R}^{2}, \mathcal{F}_{T^{k}}\right) \in \partial K_{0},
$$

where the projection operator $\mathbb{P}_{K_{0}}$ is defined in Lemma 11.3. We define $\Delta^{+} k_{T_{k}}$ by the equality

$$
\Delta^{+} V_{T_{k}}:=\gamma\left(V_{T_{k}}\right) \Delta^{+} k_{T_{k}} .
$$

Applying Theorem 11.2 and the strong markov property, there exists a solution $(\tilde{V}, \tilde{k})$ to (11.62) from the starting point $\tilde{V}_{0}:=V_{T^{k}}+$ with respect to $\tilde{N}_{t}:=N_{T^{k}+t}-N_{T^{k}}$ and $\tilde{W}_{t}:=W_{T^{k}+t}-W_{T^{k}}$ defined on the interval $\left[0, T^{k+1}-T^{k}\right]$. We then define

$$
V_{t}:=\tilde{V}_{t-T^{k}}, \text { and } k_{t}:=k_{T_{k}+}+\tilde{k}_{t-T^{k}}, \quad t \in\left(T^{k}, T^{k+1}\right) .
$$

Uniqueness follows from uniqueness on each interval $\left[T_{k}, T_{k+1}\right)$.

\section{References}

1. Alvarez O., Lasry J.-M ., and Lions P.-L. Convex viscossity solutions and states constraints. J. Math. Pures Appl., 76, 1997, p.265-288.

2. Constantinides G.M. Capital market equilibrium with transaction costs. Journal of Political Economy, 94, 1996, 842-862.

3. Davis M., Norman A. Portfolio selection with transaction costs. Math. Oper. Res., $15,1990,676-713$ 
4. De Vallière D., Kabanov Y., Lépinette E. Consumption-investment optimization problem in a Lévy financial model with transaction costs. Finance and Stochastics, 20 (2016),3, 705-740.

5. Dupuis P., Ishii H. SDEs with Oblique Reflection on Nonsmooth Domains. The Annals of Probability, Vol. 21, No. 1, Jan., 1993, pp. 554-580.

6. Framstad, N., Øksendal, B., Sulem, A.. Optimal consumption and portfolio in a jump diffusion market. Journal of Mathematical Economics, 35, 2001, 233-257.

7. Guasoni P., Lépinette E. and Rásony M. The Fundamental Theorem of Asset Pricing under Transaction Costs. Finance and Stochastics, 16, 2012, 4, 741-777.

8. Jacod J., Shiryaev A.N. Limit theorems for stochastic processes. 2.ed.-Berlin; Heidelberg; New York; Hong Kong; London; Milan; Paris; Tokyo: Springer, 2002.

9. Kabanov Yuri, Safarian M. Markets with Transaction Costs. Mathematical Theory. Monograph.

10. Merton R.C. Optimum consumption and portfolio rules in a continuous time model. J. Econ. Theory, 3, 1971, 373-413.

11. Protter P.E. Stochastic integration and differential equations. 2nde Edition 3-54000313-4 Springer-Verlag Berlin Heilderbeg New York.

12. Shreve S. E. and Soner H. M. Optimal Investment and Consumption with Transaction Costs.Ann. Appl. Probab. Volume 4, Number 3, 1994, 609-692.

13. Touzi N., Soner H.M. Dynamic programming for stochastic target problems and geometric flows. Journal of the European Mathematical Society, 4, 201-236, 2002.

14. Wheeden, R. L. Zygmund, A. Measure and integral, Marcel Dekker Inc., New York. An introduction to real analysis, Pure and Applied Mathematics, Vol. 43, 1977. 